\title{
S3-Leitlinie: Diagnostik und Therapie des hepatozellulären Karzinoms
}

\author{
Kurzversion 2.00 - Juni 2021, AWMF-Registernummer: 032-0530L
}

\section{Autoren}

Voesch Sabrina', Bitzer Michael ${ }^{1}$, Albert Jörg ${ }^{2}$, Bartenstein Peter ${ }^{3}$, Bechstein Wolf ${ }^{4}$, Blödt Susanne ${ }^{5}$, Brunner Thomas ${ }^{6}$,

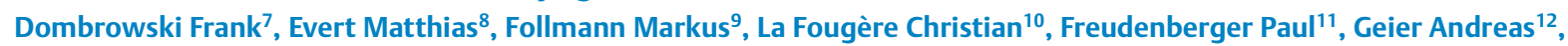
Gkika Eleni ${ }^{13}$, Götz Martin ${ }^{14}$, Hammes Elke ${ }^{15}$, Helmberger Thomas ${ }^{16}$, Hoffmann Ralf-Thorsten ${ }^{17}$, Hofmann Wolf-Peter ${ }^{18}$, Huppert Peter ${ }^{19}$, Kautz Achim ${ }^{20}$, Knötgen Gabi ${ }^{21}$, Körber Jürgen ${ }^{22}$, Krug David ${ }^{23}$, Lammert Frank ${ }^{24}$, Lang Hauke La $^{25}$, Langer Thomas ${ }^{26}$, Lenz Philipp ${ }^{27}$, Mahnken Andreas ${ }^{28}$, Meining Alexander ${ }^{29}$, Micke Oliver ${ }^{30}$, Nadalin Silvio ${ }^{31}$, Nguyen Huu Phuc $^{32}$, Ockenga Johann ${ }^{33}$, Oldhafer Karl-Jürgen ${ }^{34}$, Paprottka Philipp ${ }^{35}$, Paradies Kerstin ${ }^{36}$, Pereira Philippe ${ }^{37}$, Persigehl Thorsten ${ }^{38}$, Plauth Mathias ${ }^{39}$, Plentz Ruben ${ }^{40}$, Pohl Jürgen ${ }^{41}$, Riemer Jutta ${ }^{42}$, Reimer Peter ${ }^{43}$, Ringwald Johanna ${ }^{44}$, Ritterbusch Ulrike ${ }^{45}$, Roeb Elke ${ }^{46}$, Schellhaas Barbara ${ }^{47}$, Schirmacher Peter ${ }^{48}$, Schmid Irene ${ }^{49}$, Schuler Andreas ${ }^{50}$, von Schweinitz Dietrich ${ }^{51}$, Seehofer Daniel ${ }^{52}$, Sinn Marianne ${ }^{53}$, Stein Alexander ${ }^{54}$, Stengel Andreas ${ }^{55}$, Steubesand Nadine ${ }^{56}$, Stoll Christian ${ }^{57}$, Tannapfel Andrea ${ }^{58}$, Taubert Anne ${ }^{59}$, Trojan Jörg ${ }^{60}$, van Thiel Ingo ${ }^{61}$, Tholen Reina ${ }^{62}$, Vogel Arndt ${ }^{63}$, Vogl Thomas ${ }^{64}$, Vorwerk Hilke ${ }^{65}$, Wacker Frank ${ }^{66}$, Waidmann Oliver ${ }^{67}$, Wedemeyer Heiner ${ }^{68}$, Wege Henning ${ }^{69}$, Wildner Dane ${ }^{70}$, Wittekind Christian ${ }^{71}$, Wörns Marcus-Alexander ${ }^{72}$, Galle Peter ${ }^{72}$, Malek Nisar ${ }^{73}$

Institute

1 Medizinische Klinik I, Universitätsklinikum Tübingen, Tübingen

2 Abteilung für Gastroenterologie, Hepatologie und Endokrinologie, Stuttgart

3 Klinik und Poliklinik für Nuklearmedizin, LMU Klinikum, München

4 Klinik für Allgemein-, Viszeral-, Transplantations- und Thoraxchirurgie, Universitätsklinikum Frankfurt, Frankfurt am Main

5 AWMF-Geschäftsstelle, Berlin

6 Klinik für Strahlentherapie, Universitätsklinikum Magdeburg A. ö. R., Magdeburg

7 Institut für Pathologie, Universitätsmedizin Greifswald, Greifswald

8 Institut für Pathologie, Regensburg

9 Office des Leitlinienprogrammes Onkologie, c/o Deutsche Krebsgesellschaft e.V. Berlin

10 Nuklearmedizin und Klinische Molekulare Bildgebung, Tübingen

11 Clinical Guideline Services GmbH, Berlin

12 Medizinische Klinik und Poliklinik II, Universitätsklinikum Würzburg, Würzburg

13 Klinik für Strahlenheilkunde, Department für Radiologische Diagnostik und Therapie, Universitätsklinikum Freiburg, Freiburg

14 Kliniken Böblingen, Böblingen

15 Lebertransplantierte Deutschland e. V., Ansbach

16 Institut für Radiologie, Neuroradiologie und minimalinvasive Therapie, München Klinik Bogenhausen, München

17 Institut und Poliklinik für Diagnostische und Interventionelle Radiologie, Dresden
18 Gastroenterologie am Bayerischen Platz, medizinisches Versorgungszentrum, Berlin

19 Radiologisches Zentrum, Max Grundig Klinik, Bühl

20 Deutsche Leberhilfe e.V., Köln

21 Konferenz onkologischer Kranken- und Kinderkrankenpflege, Hamburg

22 Klinik Nahetal, Fachklinik für onkologische Rehabilitation und Anschlussrehabilitation, (AHB), Bad Kreuznach

23 Klinik für Strahlentherapie, Universitätsklinikum Schleswig-Holstein, Kiel

24 Medizinische Hochschule Hannover, Hannover

25 Klinik für Allgemein-, Viszeral und Transplantationschirurgie, Universitätsmedizin der Johannes Gutenberg-Universität Mainz, Mainz

26 Office des Leitlinienprogrammes Onkologie, c/o Deutsche Krebsgesellschaft e.V. Berlin

27 Universitätsklinikum Münster, Zentrale Einrichtung Palliativmedizin, Münster

28 Klinik für Diagnostische und Interventionelle Radiologie, Universitätsklinikum Gießen und Marburg GmbH, Marburg

29 Medizinische Klinik und Poliklinik II des Universitätsklinikums Würzburg, Würzburg

30 Klinik für Strahlentherapie und Radioonkologie, Franziskus Hospital Bielefeld, Bielefeld

31 Universitätsklinik für Allgemein-, Viszeral- und Transplantationschirurgie, Universitätsklinikum Tübingen, Tübingen

32 Humangenetik, Ruhr-Universität, Bochum

33 Medizinische Klinik II, Klinikum Bremen-Mitte, Bremen

34 Klinik für Leber-, Gallenwegs- und Pankreaschirurgie, Semmelweis Universität, Asklepios Campus Hamburg, Hamburg 
35 Abteilung für interventionelle Radiologie, Klinikum rechts der Isar der Technischen Universität München, München

36 Konferenz onkologischer Kranken- und Kinderkrankenpflege, Hamburg

37 Zentrum für Radiologie, Minimal-invasive Therapien und Nuklearmedizin, Klinikum am Gesundbrunnen, SLKKliniken Heilbronn GmbH, Heilbronn

38 Institut für Diagnostische und Interventionelle Radiologie, Universitätsklinikum Köln, Köln

39 Städtisches Klinikum Dessau, Dessau

40 Klinikum Bremen-Nord, Innere Medizin, Bremen

41 Interventionelles Endoskopiezentrum und Schwerpunkt Gastrointestinale Onkologie, Asklepios Klinik Altona, Hamburg

42 Lebertransplantierte Deutschland e. V., Bretzfeld

43 Institut für diagnostische und interventionelle Radiologie, Städtisches Klinikum Karlsruhe gGmbH, Karlsruhe

44 Psychosomatische Medizin und Psychotherapie, Universitätsklinikum Tübingen, Tübingen

45 Hospizarbeit am Universitätsklinikum Essen, Essen

46 Medizinische Klinik II, Universitätsklinikum Gießen und Marburg GmbH, Gießen

47 Medizinische Klinik I, Friedrich-Alexander Universität Erlangen-Nürnberg, Erlangen

48 Pathologisches Institut, Universitätsklinikum Heidelberg, Heidelberg

49 Zentrum Pädiatrische Hämatologie und Onkologie, Dr. von Haunersches Kinderspital, Klinikum der Universität München, München

50 Medizinische Klinik, Alb Fils Kliniken GmbH, Göppingen

51 Dietrich (SRH Wilhelm Löhe Hochschule, Fürth

52 Klinik und Poliklinik für Viszeral-, Transplantations-, Thorax- und Gefäßchirurgie, Universitätsklinikum Leipzig, Leipzig

53 Medizinische Klinik II, Universitätsklinikum HamburgEppendorf, Hamburg

54 Hämatologisch-Onkologischen Praxis Eppendorf, Hamburg

55 Psychosomatische Medizin und Psychotherapie, Universitätsklinikum Tübingen, Tübingen

56 Clinical Guideline Services $\mathrm{GmbH}$, Kiel

57 Klinik Herzoghöhe Bayreuth, Bayreuth

58 Institut für Pathologie der Ruhr-Universität Bochum am Berufsgenossenschaftlichen Universitätsklinikum Bergmannsheil, Bochum

59 Kliniksozialdienst, Universitätsklinikum Heidelberg, Bochum
60 Medizinische Klinik I, Universitätsklinikum Frankfurt, Frankfurt am Main

61 Deutsche Leberhilfe e.V., Köln

62 Deutscher Verband für Physiotherapie e. V., Köln

63 Klinik für Gastroenterologie, Hepatologie, Endokrinologie der Medizinischen Hochschule Hannover, Hannover

64 Universitätsklinikum Frankfurt, Institut für Diagnostische und Interventionelle Radiologie, Frankfurt

65 Klinik für Strahlentherapie, Universitätsklinikum Gießen und Marburg GmbH, Marburg

66 Institut für Diagnostische und Interventionelle Radiologie der Medizinischen Hochschule Hannover, Hannover

67 Medizinische Klinik I, Universitätsklinikum Frankfurt, Frankfurt am Main

68 Klinik für Gastroenterologie, Hepatologie und Endokrinologie Medizinische Hochschule Hannover, Hannover

69 I. Medizinische Klinik und Poliklinik, Universitätsklinikum Hamburg-Eppendorf, Hamburg

70 Innere Medizin, Krankenhäuser Nürnberger Land GmbH, Lauf an der Pegnitz

71 Institut für Pathologie, Universitätsklinikum Leipzig, Leipzig

72 I. Medizinische Klinik und Poliklinik, Universitätsklinikum Mainz, Mainz

73 Medizinische Klinik I, Universitätsklinikum Tübingen, Tübingen

Bibliografie

Z Gastroenterol 2022; 60: 81-107

DOI 10.1055/a-1589-7520

ISSN 0044-2771

(C) 2022. Thieme. All rights reserved.

Georg Thieme Verlag KG, Rüdigerstraße 14,

70469 Stuttgart, Germany

\section{Korrespondenzadresse}

Sabrina Voesch

Medizinische Klinik I

Universitätsklinikum Tübingen, Otfried-Müller-Str. 10,

72076 Tübingen, Deutschland

Sabrina.Voesch@med.uni-tuebingen.de

Nisar Malek

Medizinische Klinik I

Universitätsklinikum Tübingen, Otfried-Müller-Str. 10,

72076 Tübingen, Deutschland

\begin{tabular}{|l|l|l|}
\hline \multicolumn{2}{|l|}{ Inhaltsverzeichnis } & Seite \\
\hline $\begin{array}{l}\text { Wesentliche Neuerungen in der Leitlinie zur Diagnostik } \\
\text { und Therapie des hepatozellulären Karzinoms }\end{array}$ & 83 \\
\hline 1. & Informationen zu dieser Leitlinie & 84 \\
\hline 1.1. & Herausgeber & 84 \\
\hline
\end{tabular}

\begin{tabular}{|l|l|l|}
\hline \multicolumn{2}{|l|}{ Inhaltsverzeichnis } & Seite \\
\hline 1.2. & Federführende Fachgesellschaft(en) & 84 \\
\hline 1.3. & Finanzierung der Leitlinie & 84 \\
\hline 1.4. & Kontakt & 84 \\
\hline 1.5. & Zitierweise & 84 \\
\hline
\end{tabular}




\begin{tabular}{|c|c|c|}
\hline \multicolumn{2}{|c|}{ Inhaltsverzeichnis } & \multirow{2}{*}{$\begin{array}{l}\text { Seite } \\
84\end{array}$} \\
\hline 1.6. & Besonderer Hinweis & \\
\hline 1.7. & Ziele des Leitlinienprogramms Onkologie & 85 \\
\hline 1.8. & Weitere Dokumente zu dieser Leitlinie & 85 \\
\hline 1.9. & Zusammensetzung der Leitliniengruppe & 85 \\
\hline 1.9.1. & Koordination und Redaktion & 85 \\
\hline 1.9.2. & $\begin{array}{l}\text { Beteiligte Fachgesellschaften und } \\
\text { Organisationen }\end{array}$ & 85 \\
\hline 1.9.3. & Patientenbeteiligung & 86 \\
\hline 1.9.4. & Methodische Begleitung & 86 \\
\hline 1.10. & Verwendete Abkürzungen & 87 \\
\hline 2. & Einführung & 89 \\
\hline 2.1. & Geltungsbereich und Zweck & 89 \\
\hline 2.1.1. & Zielsetzung und Fragestellung & 89 \\
\hline 2.1.2. & Adressaten & 89 \\
\hline 2.1.3. & Gültigkeitsdauer und Aktualisierungsverfahren & 89 \\
\hline 2.1.4. & Grundlagen der Methodik & 89 \\
\hline 2.1.5. & $\begin{array}{l}\text { Schema der Evidenzgraduierung nach Oxford } \\
\text { (Version 2011) }\end{array}$ & 89 \\
\hline 2.1.6. & Schema der Empfehlungsgraduierung & 90 \\
\hline 2.1.7. & Statements & 90 \\
\hline 2.1.8. & Expertenkonsens (EK) & 90 \\
\hline 2.1.9. & Klug entscheiden & 90 \\
\hline 2.1.10. & $\begin{array}{l}\text { Unabhängigkeit und Darlegung möglicher } \\
\text { Interessenkonflikte }\end{array}$ & 90 \\
\hline 3. & $\begin{array}{l}\text { Diagnostik und Therapie des hepatozellulären } \\
\text { Karzinoms }\end{array}$ & 92 \\
\hline 3.1. & Risikofaktoren, Prävention und Früherkennung & 92 \\
\hline 3.1.1. & Risikofaktoren und Früherkennung & 92 \\
\hline 3.1 .2 & $\begin{array}{l}\text { Prophylaktische und therapeutische } \\
\text { Maßnahmen zur Reduktion des HCC-Risikos }\end{array}$ & 93 \\
\hline 3.1.3. & Vorsorgeuntersuchung & 93 \\
\hline 3.2. & Histopathologische und molekulare Diagnostik & 94 \\
\hline 3.3. & Bildgebende Diagnostik & 94 \\
\hline 3.3.1. & Diagnostikalgorithmus & 94 \\
\hline 3.3.2. & Staging-Systeme (Clip, TNM, Okuda, BCLC etc.) & 96 \\
\hline 3.4. & $\begin{array}{l}\text { Operative und interventionelle } \\
\text { Therapieverfahren }\end{array}$ & 96 \\
\hline 3.4.1. & Operative Therapieverfahren & 96 \\
\hline 3.4.1.1. & Lebertransplantation & 96 \\
\hline 3.4.1.1.1. & Bridging-Therapie & 97 \\
\hline 3.4.1.1.2. & Downstaging & 97 \\
\hline 3.4.1.1.3. & Nachsorge nach Lebertransplantation & 97 \\
\hline 3.4.1.2. & Resektion & 98 \\
\hline 3.4.1.2.1. & $\begin{array}{l}\text { Resektion beim hepatozellulären Karzinom } \\
\text { ohne Leberzirrhose }\end{array}$ & 98 \\
\hline 3.4.1.2.2. & $\begin{array}{l}\text { Resektion beim hepatozellulären Karzinom } \\
\text { mit Leberzirrhose }\end{array}$ & 98 \\
\hline
\end{tabular}

\begin{tabular}{|c|c|c|}
\hline \multicolumn{2}{|c|}{ Inhaltsverzeichnis } & \multirow{2}{*}{$\begin{array}{l}\text { Seite } \\
98\end{array}$} \\
\hline 3.4.2. & Interventionelle Therapieverfahren & \\
\hline 3.4.2.1. & Perkutane Ablation & 98 \\
\hline 3.4.2.2. & Transarterielle Chemoembolisation (TACE) & 98 \\
\hline 3.4.2.3. & Transarterielle Radioembolisation (TARE) & 99 \\
\hline 3.4.3. & Stereotaxie & 99 \\
\hline 3.4.4. & $\begin{array}{l}\text { Nachsorge und Erfolgskontrolle nach } \\
\text { interventioneller Therapie }\end{array}$ & 99 \\
\hline 3.4.5. & Therapiealgorithmus & 100 \\
\hline 3.5. & Systemtherapie & 101 \\
\hline 3.5.1. & $\begin{array}{l}\text { Substanzen mit einem Wirksamkeitsnachweis in } \\
\text { Phase-III-Studien für die Systemtherapie des HCC }\end{array}$ & 101 \\
\hline 3.5.2. & Medikamentöse Erstlinientherapie des HCC & 101 \\
\hline 3.5.3. & $\begin{array}{l}\text { Medikamentöse Therapie nach Versagen, bei } \\
\text { Unverträglichkeit oder bei Kontraindikationen } \\
\text { der Erstlinientherapie des HCC }\end{array}$ & 102 \\
\hline 3.5.4. & $\begin{array}{l}\text { Medikamentöse Therapie bei Leberzirrhose } \\
\text { CHILD-Pugh B/C }\end{array}$ & 103 \\
\hline 3.5.5. & $\begin{array}{l}\text { Kombination oder Vergleich von } \\
\text { Systemtherapie mit lokoregionärer Therapie }\end{array}$ & 103 \\
\hline 3.5.6. & Adjuvante Therapie nach Resektion & 103 \\
\hline 3.5.7. & Verlaufskontrollen unter Systemtherapie & 103 \\
\hline 3.5.8. & HCC bei Kindern und Jugendlichen & 103 \\
\hline 3.6. & $\begin{array}{l}\text { Supportivtherapie des hepatozellulären } \\
\text { Karzinoms und der biliären Karzinome }\end{array}$ & 104 \\
\hline 3.6.1. & Ernährung & 104 \\
\hline 3.6.2. & $\begin{array}{l}\text { Palliativmedizinische Behandlung beim } \\
\mathrm{HCC} / \mathrm{CCA}\end{array}$ & 104 \\
\hline 3.6.3. & Rehabilitation, Sport- und Bewegungstherapie & 104 \\
\hline 3.6.4. & Psychoonkologie & 104 \\
\hline 4. & Diagnostik und Therapie biliärer Karzinome & 105 \\
\hline 5. & Qualitätsindikatoren & 105 \\
\hline 6. & Abbildungsverzeichnis & 105 \\
\hline 7. & Tabellenverzeichnis & 105 \\
\hline Literatur & & 105 \\
\hline
\end{tabular}

\section{Wesentliche Neuerungen in der Leitlinie zur Diagnostik und Therapie des hepatozellulären Karzinoms}

Die S3-Leitlinie „Diagnostik und Therapie des hepatozellulären Karzinoms und der biliären Karzinome" umfasst 2 Tumorentitäten. Bisher besteht nur eine Leitlinie zur Diagnostik und Therapie des hepatozellulären Karzinoms (HCC). Daher wird in diesem Kapitel nur auf die Veränderungen der Leitlinie des hepatozellulären Karzinoms eingegangen.

Folgende wesentliche Änderungen ergeben sich zur S3-Leitlinie des hepatozellulären Karzinoms von 2013: 
Histopathologische und molekulare Diagnostik (S. 94):

Die Leberbiopsie wurde als wichtige diagnostische Maßnahme zur Diagnosestellung des hepatozellulären Karzinoms (in der zirrhotischen Leber) gestärkt. In der palliativen Situation sollte eine bioptische Sicherung erfolgen.

- Bildgebende Diagnostik (S. 94): Bisher wurde in einer zirrhotischen Leber die Diagnose eines hepatozellulären Karzinoms ab $2 \mathrm{~cm}$ mittels zweier unabhängiger Bildgebungen (MRT, CT oder Ultraschall) bei typischem Kontrastverhalten gestellt. Die Empfehlung wurde dahingehend geändert, dass die Diagnose eines hepatozellulären Karzinoms in zirrhotischer Leber aufgrund eines typischen Kontrastmittelverhaltens mit arterieller Hypervaskularisation und Auswaschen in der portalvenösen und venösen Phase im kontrastverstärkten MRT diagnostiziert werden soll. Bei unklarem MRT-Befund sollten ein triphasisches CT und/oder ein kontrastverstärkter Ultraschall herangezogen werden.

- Chirurgische Therapieverfahren (S. 96): Bei geeigneten Patienten mit einem HCC außerhalb der Mailand-Kriterien und innerhalb der UCSF-Kriterien kann eine Lebertransplantation erfolgen, insbesondere dann, wenn ein Downstaging bis innerhalb der Mailand-Kriterien gelingt. Zum Downstaging stehen, ebenso wie beim Bridging, mehrere Therapieverfahren (transarterielle Verfahren, ablative Verfahren und Leberresektion) zur Verfügung.

- Interventionelle Therapieverfahren (S.96):

- Als Standardmethode der lokalablativen Verfahren war bisher nur die Radiofrequenzablation empfohlen. Aufgrund der inzwischen guten Datenlage für die Mikrowellenablation wurde diese als äquivalentes Verfahren aufgenommen.

- Die transarterielle Chemoembolisation (TACE) sollte mehrfach durchgeführt werden, solange ein Ansprechen hierauf nachweisbar ist und behandelbare hypervaskularisierte Tumoranteile verbleiben.

- Die Indikation zur Fortführung der TACE soll nach 2 Behandlungszyklen im Tumorboard überprüft werden.

- Die transarterielle Radioembolisation (TARE) kann nach Beschluss des Tumorboards bei Patienten mit erhaltener Leberfunktion im intermediären HCC-Stadium anstelle einer TACE eingesetzt werden.

- Eine Hochpräzisionsradiotherapie (Stereotactic Body Radiotherapy, SBRT) kann in Betracht gezogen werden, wenn andere lokale Therapieverfahren nicht möglich sind (z. B. hohe Wahrscheinlichkeit für ein Therapieversagen, eingeschränkte Leberfunktion, technische Hindernisse).

- Systemtherapie (S. 101): Zum Zeitpunkt der letzten Leitlinie konnte nur Sorafenib evidenzbasiert empfohlen werden. Zwischenzeitlich sind mehrere weitere Substanzen hinzugekommen:

- Die Kombinationstherapie von Atezolizumab und Bevacizumab ist als Erstlinie zugelassen und in der Zulassungsstudie Sorafenib überlegen.

- Als weitere Erstlinientherapie ist der Tyrosinkinase-Inhibitor Lenvatinib zugelassen.

- Mehrere Tyrosinkinase-Inhibitoren und der VEGFR-R2-Antikörper Ramucirumab (bei AFP > $400 \mathrm{ng} / \mathrm{ml}$ ) stehen als weitere zugelassene Therapieoptionen zur Verfügung.

\section{Informationen zu dieser Leitlinie}

\subsection{Herausgeber}

Leitlinienprogramm Onkologie der Arbeitsgemeinschaft der Wissenschaftlichen Medizinischen Fachgesellschaften e. V. (AWMF), der Deutschen Krebsgesellschaft e. V. (DKG) und der Deutschen Krebshilfe (DKH).

\subsection{Federführende Fachgesellschaft(en)}

Deutsche Gesellschaft für Gastroenterologie, Verdauungs- und Stoffwechselkrankheiten

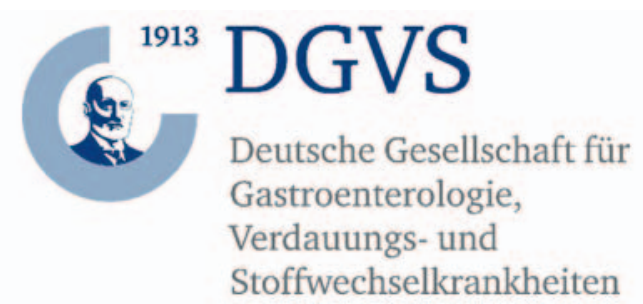

\subsection{Finanzierung der Leitlinie}

Diese Leitlinie wurde von der Deutschen Krebshilfe im Rahmen des Leitlinienprogramms Onkologie gefördert.

\subsection{Kontakt}

Office Leitlinienprogramm Onkologie c/o Deutsche Krebsgesellschaft e. V. Kuno-Fischer-Straße 8 14057 Berlin leitlinienprogramm@krebsgesellschaft.de www.leitlinienprogramm-onkologie.de

\subsection{Zitierweise}

Leitlinienprogramm Onkologie (Deutsche Krebsgesellschaft, Deutsche Krebshilfe, AWMF): Diagnostik und Therapie des hepatozellulären Karzinoms und biliärer Karziome, Langversion 2.0, Juni 2021, AWMF Registernummer: 032/-053OL, https://www.leit linienprogramm-onkologie.de/leitlinien/hepatozellulaeres-karzi nom-hcc/

\subsection{Besonderer Hinweis}

Die Medizin unterliegt einem fortwährenden Entwicklungsprozess, sodass alle Angaben, insbesondere zu diagnostischen und therapeutischen Verfahren, immer nur dem Wissensstand zur Zeit der Drucklegung der Leitlinie entsprechen können. Hinsichtlich der angegebenen Empfehlungen zur Therapie und der Auswahl sowie Dosierung von Medikamenten wurde die größtmögliche Sorgfalt beachtet. Gleichwohl werden die Benutzer aufgefordert, die Beipackzettel und Fachinformationen der Hersteller zur Kontrolle heranzuziehen und im Zweifelsfall einen Spezialisten zu konsultieren. Fragliche Unstimmigkeiten sollen bitte im allgemeinen Interesse der OL-Redaktion mitgeteilt werden. 


\section{Der Benutzer selbst bleibt verantwortlich für jede diagnostische und therapeutische Applikation, Medikation und Dosierung.}

In dieser Leitlinie sind eingetragene Warenzeichen (geschützte Warennamen) nicht besonders kenntlich gemacht. Es kann also aus dem Fehlen eines entsprechenden Hinweises nicht geschlossen werden, dass es sich um einen freien Warennamen handelt.

Das Werk ist in allen seinen Teilen urheberrechtlich geschützt. Jede Verwertung außerhalb der Bestimmung des Urhebergesetzes ist ohne schriftliche Zustimmung der OL-Redaktion unzulässig und strafbar. Kein Teil des Werkes darf in irgendeiner Form ohne schriftliche Genehmigung der OL-Redaktion reproduziert werden. Dies gilt insbesondere für Vervielfältigungen, Übersetzungen, Mikroverfilmungen und die Einspeicherung, Nutzung und Verwertung in elektronischen Systemen, Intranets und dem Internet.

In dieser Leitlinie wird aus Gründen der Lesbarkeit die männliche Form verwendet, nichtsdestotrotz beziehen sich die Angaben auf Angehörige aller Geschlechter.

\subsection{Ziele des Leitlinienprogramms Onkologie}

Die Arbeitsgemeinschaft der Wissenschaftlichen Medizinischen Fachgesellschaften e. V., die Deutsche Krebsgesellschaft e. V. und die Deutsche Krebshilfe haben sich mit dem Leitlinienprogramm Onkologie (OL) das Ziel gesetzt, gemeinsam die Entwicklung und Fortschreibung und den Einsatz wissenschaftlich begründeter und praktikabler Leitlinien in der Onkologie zu fördern und zu unterstützen. Die Basis dieses Programms beruht auf den medizinisch-wissenschaftlichen Erkenntnissen der Fachgesellschaften und der DKG, dem Konsens der medizinischen Fachexperten, Anwender und Patienten sowie auf dem Regelwerk für die Leitlinienerstellung der AWMF und der fachlichen Unterstützung und Finanzierung durch die Deutsche Krebshilfe. Um den aktuellen Stand des medizinischen Wissens abzubilden und den medizinischen Fortschritt zu berücksichtigen, müssen Leitlinien regelmäßig überprüft und fortgeschrieben werden. Die Anwendung des AWMF-Regelwerks soll hierbei Grundlage zur Entwicklung qualitativ hochwertiger onkologischer Leitlinien sein. Da Leitlinien ein wichtiges Instrument der Qualitätssicherung und des Qualitätsmanagements in der Onkologie darstellen, sollten sie gezielt und nachhaltig in den Versorgungsalltag eingebracht werden. So sind aktive Implementierungsmaßnahmen und auch Evaluationsprogramme ein wichtiger Bestandteil der Förderung des Leitlinienprogramms Onkologie. Ziel des Programms ist es, in Deutschland professionelle und mittelfristig finanziell gesicherte Voraussetzungen für die Entwicklung und Bereitstellung hochwertiger Leitlinien zu schaffen. Diese hochwertigen Leitlinien dienen nicht nur dem strukturierten Wissenstransfer, sondern können auch in der Gestaltung der Strukturen des Gesundheitssystems ihren Platz finden. Zu erwähnen sind hier evidenzbasierte Leitlinien als Grundlage zum Erstellen und Aktualisieren von Disease-Management-Programmen oder die Verwendung von aus Leitlinien extrahierten Qualitätsindikatoren im Rahmen der Zertifizierung von Organtumorzentren.

\subsection{Weitere Dokumente zu dieser Leitlinie}

Bei diesem Dokument handelt es sich um die Kurzversion der S3Leitlinie „Diagnostik und Therapie des hepatozellulären Karzinoms und biliärer Karzinome“. Neben der Kurzversion wird es folgende ergänzende Dokumente zu dieser Leitlinie geben:

- Langversion der Leitlinie

- Laienversion (Patientenleitlinie)

- Leitlinienreport zum Erstellungsprozess der Leitlinie

- Evidenztabellen

Diese Leitlinie und alle Zusatzdokumente sind über die folgenden Seiten zugänglich.

- Leitlinienprogramm Onkologie (https://www.leitlinienpro gramm-onkologie.de/leitlinien/hepatozellulaeres-karzinom-hcc/)

- AWMF (www.leitlinien.net)

- Deutsche Gesellschaft für Gastroenterologie, Verdauungs- und Stoffwechselkrankheiten (www.dgvs.de)

- Guidelines International Network (www.g-i-n.net)

Die Leitlinie ist außerdem in der App des Leitlinienprogramms Onkologie enthalten.

Weitere Informationen unter: https://www.leitlinienpro gramm-onkologie.de/app/.

\subsection{Zusammensetzung der Leitliniengruppe}

\subsubsection{Koordination und Redaktion}

Prof. Dr. Nisar P. Malek

Ärztlicher Direktor, Medizinische Klinik Universitätsklinikum Tübingen

Prof. Dr. Michael Bitzer

Stellvertretener Ärztlicher Direktor, Medizinische Klinik Universitätsklinikum Tübingen

Prof. Dr. Peter R. Galle

Ärztlicher Direktor, Universitätsmedizin der Johannes-GutenbergUniversität Mainz

\section{Sabrina Voesch}

Ärztin in Weiterbildung, Medizinische Klinik Universitätsklinikum Tübingen

\subsubsection{Beteiligte Fachgesellschaften und Organisationen}

In $\triangleright$ Tab. 1 sind die an der Leitlinienerstellung beteiligten medizinischen Fachgesellschaften und sonstigen Organisationen sowie deren mandatierte Vertreter aufgeführt.

Außerdem wurden folgende Fachgesellschaften für den Leitlinienprozess angeschrieben, diese haben jedoch keinen Mandatsträger benannt:

- Deutsche Gesellschaft für Allgemeinmedizin und Familienmedizin

- Deutsche Gesellschaft für Pflegewissenschaft e. V.

- Arbeitsgemeinschaft Pädiatrische Onkologie

- Arbeitsgemeinschaft Onkologische Thoraxchiurgie

- Deutsche Gesellschaft für Ernährung e. V.

- Deutsche Gesellschaft für Klinische Chemie und Laboratoriumsmedizin. 
Die Zuordnung der beteiligten Mandatsträger und Experten finden Sie in den $>$ Tab. 2, 3.

\subsubsection{Patientenbeteiligung}

Die Leitlinie wurde unter direkter Beteiligung von 4 Patientenvertretern erstellt.

Herr Ingo van Thiel, Herr Achim Kautz und Frau Elke Hammes waren von Beginn an in die Erstellung der Leitlinie eingebunden und nahmen mit eigenem Stimmrecht an der Konsensuskonferenz teil. Herr Kautz war der Stellvertreter von Herrn Thiel und hat daher nicht abgestimmt. Frau Riemer ersetzte Frau Hammes ab der Video-Konsensuskonferenz 08/2020.

\subsubsection{Methodische Begleitung}

1. Durch das Leitlinienprogramm Onkologie:

a) Dr. med. Markus Follmann, MPH, MSc (OL Office c/o Deutsche Krebsgesellschaft)

b) Thomas Langer, Dipl. Soz. Wiss. (OL Office c/o Deutsche Krebsgesellschaft)

2. Durch die Arbeitsgemeinschaft der Wissenschaftlichen Medizinischen Fachgesellschaften e. V.:

a) Dr. rer. medic. Susanne Blödt, MScPH (AWMF-IMWI)

3. Durch die Firma Clinical Guideline Service - User Group:

a) Dr. Nadine Steubesand

b) Dr. Paul Freudenberger

4. Durch die DGVS

a) Pia Lorenz

b) PD Dr. med. Petra Lynen Jansen

- Tab. 1 Beteiligte Fachgesellschaften und Organisationen.

\section{beteiligte Fachgesellschaften und Organisationen}

Deutsche Gesellschaft für Allgemein- und Viszeralchirurgie (DGAV)

Deutsche Gesellschaft für Chirurgie (DGCH)

Deutsche Gesellschaft für Ernährungsmedizin (DGEM)

Deutsche Gesellschaft für Endoskopie und Bildgebende Verfahren (DGE-BV)

Deutsche Gesellschaft für Hämatologie und Onkologie (DGHO)

Deutsche Gesellschaft für Innere Medizin (DGIM)

Deutsche Gesellschaft für Interventionelle Radiologie und Minimalinvasive Therapie (DeGIR)

Deutsche Gesellschaft für Nuklearmedizin (DGN)

Deutsche Gesellschaft für Pädiatrische Onkologie und Hämatologie (GPOH)

Deutsche Gesellschaft für

Palliativmedizin (DGPall)

\section{Mandatsträger}

Oldhafer K., Seehofer D. (Stellv.)

Lang $\mathrm{H}$.

Plauth M., Ockenga J.

(Stellv.)

Meining A.

Sinn M.

Bitzer M., Trojan J.

Pereira P., Mahnken A.

(Stellv.), Huppert P.

(Stellv.)

Bartenstein P., La Fougère Ch. (Stellv.)

Schmid I., v. Schweinitz D. (Stellv.)

Lenz P.

- Tab. 1 (Fortsetzung)

beteiligte Fachgesellschaften und Organisationen

Deutsche Gesellschaft für Pathologie (DGP)

Berufsverband deutscher Pathologen (BDP)

Deutsche Gesellschaft für

Radioonkologie (DEGRO)

Deutsche Gesellschaft für Ultraschall in der Medizin (DEGUM)

Deutscher Verband für Physiotherapie (ZKV)

Deutsche Röntgengesellschaft e. V. (DRG)

Deutsche Gesellschaft für Gastroenterologie, Verdauungs- und Stoffwechselkrankheiten (DGVS)

Deutsche Transplantationsgesellschaft (DTG)

Deutsche Gesellschaft für Humangenetik (GfH)

Deutsche Leberhilfe e.V.

\section{Deutsche Leberstiftung}

Lebertransplantierte Deutschland e. V.

Arbeitsgemeinschaft Internistische

Onkologie (AIO)

Arbeitsgemeinschaft Onkologische Pathologie

Arbeitsgemeinschaft Radiologische Onkologie (ARO)

Arbeitsgemeinschaft Tumorklassifikation in der Onkologie (ATO)

Arbeitsgemeinschaft Supportive Maßnahmen in der Onkologie (AGSMO)

Arbeitsgemeinschaft Onkologische Rehabilitation und Sozialmedizin (AGORS)

Chirurgische Arbeitsgemeinschaft Onkologie (CAO-V)

Arbeitsgemeinschaft Psychoonkologie (PSO)

Konferenz Onkologischer Krankenund Kinderkrankenpflege (KOK)

Arbeitsgemeinschaft Palliativmedizin (APM)

Arbeitsgemeinschaft Bildgebung in der Onkologie (ABO)

Arbeitsgemeinschaft Prävention und Integrative Medizin in der Onkologie (AG PRIO)

Arbeitsgemeinschaft Soziale Arbeit in der Onkologie (ASO)

\section{Mandatsträger}

Schirmacher P., Evert M.

Schirmacher P., Evert M.

Vorwerk, H., Krug D.

(Stellv.)

Schuler, A., Wildner D. (Stellv.)

Tholen, R.

Vogl, T., Paprottka P. Wacker F., Helmberger $T$.

Malek N., Galle P., Götz M., Lammert F., Plentz R.

Nadalin S.

Nguyen H.P.

van Thiel I., Kautz A. (Stellv.)

Schirmacher $P$.

Hammes E. bis

10.08.2020

Riemer J. ab 11.08.2020

Vogel A., Wege H.

Dombrowski F.

Brunner T., Gkika E. (Stellv.)

Tannapfel A., Wittekind Ch. (Stellv.)

Stein A.

Körber J.

Bechstein W.

Stengel, A., Ringwald J. (Stellv.)

Knötgen G., Paradies K. (Stellv.)

Ritterbusch U.

Persigehl Th.

Stoll Ch., Micke O. (Stellv.)

Taubert A. 
- Tab. 2 Arbeitsgruppen und deren Mitglieder.

\section{Arbeitsgruppe \\ Mitglieder der Arbeitsgruppe (AG-Leiter hervorgehoben)}

AG I. Risikofaktoren, Prävention und Früherkennung

Trojan J. , Schuler A., van Thiel I., Kautz A., Wedemeyer H., Lammert F., Roeb E., Geier A., Wildner D., Hofmann, W.P., Schmid I.

AG II.I Histopathologische und molekulare Diagnostik

AG II.II Bildgebende Diagnostik

AG III.I Operative und interventionelle Therapieverfahren

AG III.II Systemtherapie

AG IV Supportivtherapie
Schirmacher P , Nguyen H.P., Dombrowski F., Evert M., Tannapfel A.

Götz M. , Paprottka P., Persigehl T., Vogl T.J., Meining A., Wacker F., Pohl J., Reimer P., Schellhaas B.

Nadalin S., Pereira P. , Bechstein W., Oldhafer K., Lang H., Bartenstein P., Vorwerk H., Brunner T., Hammes E., Vogel A., Helmberger T., Seehofer D., La Fougère Ch., Albert J., Hoffmann R.-T., Mahnken A., Huppert P., Gkika E., Krug D.

Bitzer M., Galle P. , Sinn M., Stein A., Plentz R., Schmid I., Wörns M.-A., Wege H.

Lenz P. , Ritterbusch U., Tholen R., Körber J., Stoll Ch., Taubert A., Stengel A., Knötgen G., Plauth M., Waidmann O.

- Tab. 3 Beteiligte Experten.

\begin{tabular}{l|l}
$\begin{array}{l}\text { beteiligte } \\
\text { Experten }\end{array}$ & Arbeitsgruppe \\
\hline Wedemeyer, H. & AG I Risikofaktoren, Prävention und Früherkennung \\
\hline $\begin{array}{l}\text { Roeb, E. } \\
\text { Geier, A. }\end{array}$ & AG I Risikofaktoren, Prävention und Früherkennung \\
\hline Pohl, J. & AG I Risikofaktoren, II Biävention und Früherkennung \\
\hline Reimer, P. & AG II. II Bildgebende Diagnostik \\
\hline Schellhaas B. & AG II. II Bildgebende Diagnostik \\
\hline Albert, J. & $\begin{array}{l}\text { AG III.I. Operative und interventionelle } \\
\text { Therapieverfahren }\end{array}$ \\
\hline Hoffmann, R.-T. & $\begin{array}{l}\text { AG III.I. Operative und interventionelle } \\
\text { Therapieverfahren }\end{array}$ \\
\hline Hofmann, W. P. & AG I Risikofaktoren, Prävention und Früherkennung \\
\hline Wörns, M.-A. & AG III.II. Systemtherapie \\
\hline Waidmann, O. & AG IV. Supportivtherapie \\
\hline
\end{tabular}

\subsection{Verwendete Abkürzungen}

\begin{tabular}{|l|l|}
\hline Abkürzung & Erläuterung \\
\hline 3D-CRT & Three-dimensional Conformal Radiation Therapy \\
\hline 5 -FU & 5-Fluorouracil \\
\hline AASLD & American Association for the Study of Liver Diseases \\
\hline ACG & American College of Gastroenterology \\
\hline
\end{tabular}

\begin{tabular}{|c|c|}
\hline Abkürzung & Erläuterung \\
\hline AFIP & Armed Forces Institute of Pathology \\
\hline AFP & Alpha-Fetoprotein \\
\hline AFP-L3 & Lektin-reaktives Alpha-Fetoprotein \\
\hline AG & Arbeitsgruppe \\
\hline ALT & Alanin-Aminotransferase \\
\hline APASL & Asian Pacific Association for the Study of the Liver \\
\hline APRI & AST/Thrombozyten-Ratio-Index \\
\hline ARFI & Acoustic Radiation Force Impulse Imaging \\
\hline AST & Aspartat-Aminotransferase \\
\hline ATG & Antithymozytenglobulin \\
\hline AUC & Area under the Curve \\
\hline AWMF & $\begin{array}{l}\text { Arbeitsgemeinschaft der Wissenschaftlichen } \\
\text { Medizinischen Fachgesellschaften e. V. }\end{array}$ \\
\hline ÄZQ & Ärztliches Zentrum für Qualität in der Medizin \\
\hline BÄK & Bundesärztekammer \\
\hline BCLC & Barcelona Clinic Liver Cancer \\
\hline Bds & beiderseits \\
\hline BillN & biliäre intraepitheliale Neoplasie \\
\hline BMI & Body-Mass-Index \\
\hline BSC & Best Supportive Care \\
\hline BSG & British Society of Gastroenterology \\
\hline CA 19-9 & Carbohydrate-Antigen 19-9 \\
\hline CCA & Cholangiokarzinom \\
\hline iCCA & intrahepatisches Cholangiokarzinom \\
\hline dCCA & distales Cholangiokarzinom \\
\hline eCCA & extrahepatisches Cholangiokarzinom \\
\hline pCCA & perihiläres Cholangiokarzinom \\
\hline$C D$ & Cluster of Differentiation \\
\hline CECT & kontrastmittelverstärkte Computertomografie \\
\hline CEUS & Kontrastmittelultraschall \\
\hline $\mathrm{Cl}$ & Konfidenzintervall \\
\hline CIPN & Chemotherapie-induzierte periphere Neuropathie \\
\hline CLIP & Cancer of the Liver Italian Program \\
\hline CNI & Calcineurin-Inhibitor \\
\hline$C R$ & Complete Remission \\
\hline CT & Computertomografie \\
\hline CTCEA & Common Terminology Criteria for Adverse Events \\
\hline CTLA-4 & Cytotoxic-T-lymphocyte-associated-Protein 4 \\
\hline CU-HCC & Chinese University-HCC (Risikoscore) \\
\hline CUP & Cancer of Unkown Primary \\
\hline DAAD & Direct-acting antiviral Drugs \\
\hline DCP & des-Gamma-Carboxyprothrombin \\
\hline DGCH & Deutsche Gesellschaft für Chirurgie \\
\hline DGEM & Deutsche Gesellschaft für Ernährungsmedizin \\
\hline DGVS & $\begin{array}{l}\text { Deutsche Gesellschaft für Gastroenterologie, } \\
\text { Verdauungs- und Stoffwechselkrankheiten }\end{array}$ \\
\hline
\end{tabular}




\begin{tabular}{|c|c|c|c|}
\hline Abkürzung & Erläuterung & Abkürzung & Erläuterung \\
\hline $\mathrm{DHC}$ & Ductus choledochus & IGRT & Image Guided Radiotherapy \\
\hline DKG & Deutsche Krebsgesellschaft e. V. & ILCA & International Liver Cancer Association \\
\hline DKH & Deutsche Krebshilfe e. V. & IPMN & intraduktale papillär-muzinöse Neoplasie \\
\hline EASL & European Association for the Study of the Liver & IRE & irreversible Elektroporation \\
\hline ECOG & Eastern Cooperative Oncology Group & \multirow[t]{2}{*}{ IQWiG } & \multirow{2}{*}{$\begin{array}{l}\text { Institut für Qualität und Wirtschaftlichkeit } \\
\text { im Gesundheitswesen }\end{array}$} \\
\hline \multirow[t]{2}{*}{ EG } & \multirow{2}{*}{$\begin{array}{l}\text { Empfehlungsgrad, } A=\text { starke Empfehlung, } \\
B=\text { Empfehlung, } 0=\text { offene Empfehlung }\end{array}$} & & \\
\hline & & ITT & Intention to Treat \\
\hline EK & Expertenkonsens & JIS & Japan Integrated Staging Score \\
\hline ELTR & European Liver Transplant Registry & KASL & Korean Association for the Study of the Liver \\
\hline \multirow[t]{2}{*}{ EORTC } & \multirow{2}{*}{$\begin{array}{l}\text { European Organisation for Research and Treatment } \\
\text { of Cancer }\end{array}$} & LA & Leitlinienadaptation \\
\hline & & \multirow[t]{2}{*}{ LAASL } & \multirow{2}{*}{$\begin{array}{l}\text { Latin American Association for the Study of the Liver } \\
\text { (LAASL) }\end{array}$} \\
\hline \multirow[t]{2}{*}{ EORTIC-QLQ } & \multirow{2}{*}{$\begin{array}{l}\text { European Organisation for Research and Treatment } \\
\text { of Cancer - Quality of Life Questionnaire }\end{array}$} & & \\
\hline & & LiMax & Maximum liver function capacity \\
\hline EQD2 & Äquivalentdosis von 2 Gy & LiRADS & Liver Imaging Reporting and Data System \\
\hline $\mathrm{ERC}(\mathrm{P})$ & endoskopische retrograde Cholangio(pankreatiko)grafie & $\mathrm{LL}$ & Leitlinie \\
\hline ESCULAP & $\begin{array}{l}\text { Erlanger Synopsis for Contrast-enhanced Ultrasound } \\
\text { for Liver Lesion Assessment in Patients at Risk }\end{array}$ & LoE & Level of Evidence \\
\hline ESMO & European Society of Medical Oncology & LTx & Lebertransplantation \\
\hline ETC & Extended Toronto Criteria & MARS & Molecular-Adsorbent-Recirculating-System \\
\hline EUS-(FNA) & endosonografischer Ultraschall - (Feinnadelaspiration) & MCN & muzinös-zystische Neoplasie \\
\hline FACT-H & Functional Assessment of Cancer Therapy- Hepatobiliary & MRCP & Magnetresonanz-Cholangiopankreatikografie \\
\hline FDG-PET & Fluordeoxyglukose-Positronen-Emissionstomografie & $\operatorname{MR}(\mathrm{T})$ & Magnetresonanz(tomografie) \\
\hline FIB-4 & Fibrosis-4 & MWA & Mikrowellenablation \\
\hline \multirow{2}{*}{ GAG-HCC } & \multirow{2}{*}{$\begin{array}{l}\text { Guide with Age, Gender, HBV DNA, Core Promoter } \\
\text { Mutations and Cirrhosis-HCC }\end{array}$} & NAFLD & Non-alcoholic fatty Liver Disease \\
\hline & & $\mathrm{NASH}$ & nichtalkoholische Steatohepatitis \\
\hline GB-CA & Gallenblasenkarzinom & $\mathrm{NCl}$ & National Cancer Institute \\
\hline Gd-DTPA & Gadolinium-Diethylene-Triamine Pentaacetic Acid & NCCN & The National Comprehensive Cancer Network \\
\hline \multirow{2}{*}{$\begin{array}{l}\text { Gd-EOB- } \\
\text { DTPA }\end{array}$} & \multirow{2}{*}{$\begin{array}{l}\text { Gadolinium-Ethoxybenzyl-Diethylentriamin-Penta- } \\
\text { Essigsäure }\end{array}$} & NGC & National Guideline Clearinghouse \\
\hline & & NICE & National Institute for Health and Clinical Excellence \\
\hline GEKID & $\begin{array}{l}\text { Gesellschaft der epidemiologischen Krebsregister } \\
\text { in Deutschland e.V. }\end{array}$ & NRS & Nutrition Risk Screening \\
\hline G-I-N & Guidelines International Network & NT & nicht transplantabel \\
\hline GLOBOCAN & Global Cancer Incidence, Mortality and Prevalence & $\mathrm{OL}$ & Leitlinienprogramm Onkologie \\
\hline GOT & Glutamat-Oxalacetat-Transaminase & $\mathrm{OP}$ & Operation \\
\hline GPT & Glutamat-Pyruvat-Transaminase & ORN & Osteoradionekrose \\
\hline $\mathrm{HAl}$ & hepatische arterielle Infusion & OS & Overall Survival \\
\hline $\mathrm{HBsAg}$ & Hepatitis-B-surface-Antigen & PBC & primär biliäre Zirrhose \\
\hline $\mathrm{HBeAg}$ & Hepatitis-B-envelope-Antigen & PBD & präoperative biliäre Drainage \\
\hline HBV & Hepatitis B & PD & progressive Disease \\
\hline $\mathrm{HCC}$ & hepatozelluläres Karzinom & PDT & photodynamische Therapie \\
\hline $\mathrm{HCV}$ & Hepatitis C & PEI/PAI & perkutane Ethanol-Injektion \\
\hline HepPar1 & Hepatocyte Paraffin 1 & PET & Positronen-Emissions-Tomografie \\
\hline HR & Hazard Ratio & PFS & Progression Free Survival \\
\hline HSP70 & Hitzeschockprotein 70 & PICO & Population Intervention Comparison Outcome \\
\hline hTERT & Human Telomerase Reverse Transcriptase & PR & Partial Remission \\
\hline ICCR & International Collaboration on Cancer Reporting & PS & Progressive Disease \\
\hline ICD & International Statistical Classification of Diseases & PSC & primär sklerosierende Cholangitis \\
\hline & and Related Health Problems & PV & Portalvene \\
\hline
\end{tabular}




\begin{tabular}{|c|c|}
\hline Abkürzung & Erläuterung \\
\hline PZK & patientenzentrierte Kommunikation \\
\hline QI & Qualitätsindikatoren \\
\hline QoL & Quality of Life \\
\hline RFA & Radiofrequenzablation \\
\hline iRFA & intraduktale Radiofrequenzablation \\
\hline RILD & Radiation induced Liver Disease \\
\hline RR & relatives Risiko \\
\hline SBRT & Stereotactic Body Radiotherapy \\
\hline SD & Stable Disease \\
\hline SEOM & Spanish Society of Medical Oncology \\
\hline SEMS & selbstexpandierender Metallstent \\
\hline SGA & Subjective Global Assessment \\
\hline SGB & Sozialgesetzbuch \\
\hline SR & systematische Recherche \\
\hline STIKO & Ständige Impfkommission \\
\hline SVR & Substained Virological Response \\
\hline TACE & transarterielle Chemoembolisation \\
\hline DEB-TACE & Drug-eluting Bead TACE \\
\hline TARE & transarterielle Radioembolisation \\
\hline TNM & Tumor-Nodus-Metastase \\
\hline TTD & Time to Deterioration \\
\hline TTP & Time to Progression \\
\hline UICC & Union for International Cancer Control \\
\hline UCSF & University of California, San Francisco \\
\hline UNOS & United Network of Organ Sharing \\
\hline US & Ultraschall \\
\hline $\operatorname{VEGF(R)}$ & Vascular Endothelial Growth Factor (Receptor) \\
\hline WHO & World Health Organisation \\
\hline
\end{tabular}

\section{Einführung}

\subsection{Geltungsbereich und Zweck}

\subsubsection{Zielsetzung und Fragestellung}

Die interdisziplinäre S3-Leitlinie ist ein Instrument, um die Diagnostik und Therapie des hepatozellulären Karzinoms (HCC), des Cholangiokarzinoms (CCA) und des Gallenblasenkarzinoms zu verbessern. Fachgruppen aller Disziplinen, die Patienten mit chronischen Lebererkrankungen, Verdacht auf oder bereits diagnostizierten hepatobiliären Tumoren ambulant und/oder stationär behandeln, sollen durch die Leitlinie unterstützt werden. Die Leitlinie soll dazu beitragen, eine angemessene Gesundheitsversorgung dieser Patientengruppen sicherzustellen. Es ist weiterhin die Aufgabe der Leitlinie, betroffenen Patienten angemessene, wissenschaftlich begründete und aktuelle Verfahren in der Diagnostik, Therapie und Rehabilitation anzubieten. Dies gilt sowohl für lokal begrenzte oder lokal fortgeschrittene Erkrankungen als auch bei Vorliegen eines Rezidivs oder von Fernmetastasen. Die Leitlinie soll neben dem Beitrag für eine angemessene Gesundheitsversorgung auch die Basis für eine individuell zugeschnittene, qualitativ hochwertige und kosteneffiziente Therapie bieten. Mittel- und langfristig sollen so die Morbidität und Mortalität von Patienten mit hepatobiliären Tumoren gesenkt und die Lebensqualität erhöht werden.

\subsubsection{Adressaten}

Die Anwenderzielgruppe sind Ärztinnen und Ärzte aller Versorgungsbereiche (insbesondere Fachärztinnen und Fachärzte), die Patienten mit chronischen Lebererkrankungen, Verdacht auf sowie diagnostizierten hepatobiliären Tumoren ambulant und/ oder stationär behandeln. Hierzu gehören unter anderem Allgemeinmediziner, Internisten, Gastroenterologen und Hepatologen, Onkologen, Radiologen, Chirurgen, Palliativmediziner, Pathologen, Nuklearmediziner und Strahlentherapeuten, Psychoonkologen, onkologisch tätige Pflegekräfte und Physiotherapeuten sowie alle an einem HCC oder biliären Karzinom erkrankten Patienten und deren Angehörige. Weitere Adressaten dieser Leitlinie sind übergeordnete Organisationen (z. B. Krankenkassen). Sie soll entsprechend der Definition einer Leitlinie Entscheidungshilfen geben, jedoch keine Richtlinie sein. Der behandelnde Arzt ist weiterhin verpflichtet, unter Würdigung der Gesamtsituation des Patienten und mit diesem gemeinsam die für die individuelle Situation angemessene Vorgehensweise zu finden.

\subsubsection{Gültigkeitsdauer und Aktualisierungsverfahren}

Die S3-Leitlinie ist bis zur nächsten Aktualisierung gültig, die Gültigkeitsdauer wird auf 3 Jahre geschätzt. Vorgesehen sind regelmäßige Aktualisierungen, bei dringendem Änderungsbedarf werden diese gesondert publiziert. Kommentare und Hinweise für den Aktualisierungsprozess sind ausdrücklich erwünscht und können an die folgende Adresse gesendet werden: hcc@leitlinienprogramm-onkologie.de.

\subsubsection{Grundlagen der Methodik}

Die methodische Vorgehensweise bei der Erstellung der Leitlinie ist im Leitlinienreport dargelegt. Dieser ist im Internet z. B. auf den Seiten des Leitlinienprogramms Onkologie (http://leitlinien programm-onkologie.de/Leitlinien.7.0.html) und den Seiten der AWMF (http://www.awmf.org/) frei verfügbar.

\subsubsection{Schema der Evidenzgraduierung nach Oxford (Version 2011)}

Zur Klassifikation des Verzerrungsrisikos der identifizierten Studien wurde in dieser Leitlinie das in $>$ Tab. 4 aufgeführte System des Oxford Center for Evidence-based Medicine in der Version von 2011 verwendet. Dieses System sieht die Klassifikation der Studien für verschiedene klinische Fragestellungen (Nutzen von Therapie, prognostische Aussagekraft, diagnostische Wertigkeit) vor. 


\subsubsection{Schema der Empfehlungsgraduierung}

Die Methodik des Leitlinienprogramms Onkologie sieht eine Vergabe von Empfehlungsgraden durch die Leitlinienautoren im Rahmen eines formalen Konsensusverfahrens vor. Dementsprechend wurden durch die AWMF moderierte, nominale Gruppenprozesse bzw. strukturierte Konsensuskonferenzen durchgeführt [2]. Im Rahmen dieser Prozesse wurden die Empfehlungen von den stimmberechtigten Mandatsträgern (siehe Kapitel 1.9.2) formal abgestimmt. Die Ergebnisse der jeweiligen Abstimmungen (Konsensstärke) sind entsprechend den Kategorien in $>$ Tab. 6 den Empfehlungen zugeordnet.

In der Leitlinie werden zu allen evidenzbasierten Statements (siehe Kapitel 2.2.3) und Empfehlungen das Evidenzlevel der zugrunde liegenden Studien sowie bei Empfehlungen zusätzlich die Stärke der Empfehlung (Empfehlungsgrad) ausgewiesen. Hinsichtlich der Stärke der Empfehlung werden in dieser Leitlinie 3 Empfehlungsgrade unterschieden (siehe - Tab.5), die sich auch in der Formulierung der Empfehlungen jeweils widerspiegeln.

Die Entscheidungskriterien für die Festlegung der Empfehlungsgrade werden im Leitlinienreport zu dieser Leitlinie erläutert.

\subsubsection{Statements}

Als Statements werden Darlegungen oder Erläuterungen von spezifischen Sachverhalten oder Fragestellungen ohne unmittelbare Handlungsaufforderung bezeichnet. Sie werden entsprechend der Vorgehensweise bei den Empfehlungen im Rahmen eines formalen Konsensusverfahrens verabschiedet und können entweder auf Studienergebnissen oder auf Expertenmeinungen beruhen.

\subsubsection{Expertenkonsens (EK)}

Statements/Empfehlungen, für die eine Bearbeitung auf der Grundlage von Expertenkonsens der Leitliniengruppe beschlossen wurde, sind als Expertenkonsens ausgewiesen. Für diese Empfehlungen wurde keine systematische Literaturrecherche durchgeführt (die in den Hintergrundtexten ggf. angeführten Studien wurden von den beteiligten Fachexperten ausgewählt). Bei Empfehlungen, die auf einem Expertenkonsens basieren, werden keine Symbole bzw. Buchstaben verwendet, um die Empfehlungsstärke und die Qualität der Evidenz darzustellen. Die Stärke der Empfehlung ergibt sich hier allein aus der verwendeten Formulierung (soll/sollte/kann) entsprechend der Abstufung in $>$ Tab. $\mathbf{5}$.

\subsubsection{Klug entscheiden}

Empfehlungen, die mit „Klug entscheiden“ gekennzeichnet sind, wurden für die „Klug entscheiden“-Initiative der Deutschen Gesellschaft für Innere Medizin ausgewählt. Diese Empfehlungen sollen als konkrete Hilfestellung bei der Indikationsstellung zu diagnostischen und therapeutischen Maßnahmen dienen, um eine Unter- bzw. Überversorgung zu vermeiden. Weitere Informationen finden Sie unter https://www.klug-entscheiden.com/.

\subsubsection{Unabhängigkeit und Darlegung möglicher Interessenkonflikte}

Die Deutsche Krebshilfe stellte die finanziellen Mittel über das Leitlinienprogramm Onkologie (OL) zur Verfügung. Diese Mittel wurden eingesetzt für Personalkosten, Büromaterial, Literaturbeschaffung und die Konsensuskonferenzen (Raummieten, Technik, Verpflegung, Moderatorenhonorare, Reisekosten der Teilnehmer). Die Erarbeitung der Leitlinie erfolgte in redaktioneller Unabhängigkeit von der finanzierenden Organisation. Alle Mitglieder legten während des Leitlinienprozesses mittels des AWMF-Formblatts eine schriftliche Erklärung zu eventuell bestehenden Interessenkonflikten (zu Beginn, Aktualisierung vor der Konsenskonferenz) vor. Die Interessenerklärungen sind im Leitlinienreport zu dieser Leitlinie (https://www.leitlinienprogramm-onkologie.de/ leitlinien/hepatozellulaeres-karzinom-hcc/) aufgeführt.

Der Umgang mit Interessenkonflikten wurde analog den Vorgaben der AWMF gemeinsam in unserem Steuergruppentreffen vom 18.07.2019 konsentiert. Zusätzlich wurde dieses Vorgehen mit Vertretern der DKG und der AWMF besprochen. Hier wurde folgendes Vorgehen festgehalten:

- Es wurden alle direkten finanziellen und indirekten sekundären Interessen der letzten 3 Jahre im Formular der Interessenkonflikte angegeben. Unmittelbar vor den beiden Konsensuskonferenzen erfolgte eine Aktualisierung der Erklärung.

- Entscheidend für die Bewertung war der thematische Bezug zur Leitlinie.

- Die Erklärungen zu den Interessenkonflikten werden im Leitlinienreport aufgeführt.

Umgang mit direkten finanziellen Interessenkonflikten:

- Es wurde bei finanziellen Vergütungen durch Ad-Board, Beratertätigkeit, Vortragstätigkeit und Industriedrittmittel in verantwortlicher Position ein finanzieller Rahmen von $5000 € / J a h r$ und Firma festgesetzt. Oberhalb dieser Grenze kam es zur Feststellung eines moderaten Interessenkonfliktes.

- Wurde ein moderater Interessenkonflikt festgestellt, enthielt sich der Mandatsträger bei einzelnen Fragen oder Themenbereichen, je nach festgestelltem Interessenkonflikt.

- Im Hinblick auf die AG-Leiter wurde darauf geachtet, dass einem Mitglied der Steuergruppe mit einem moderateren Interessenkonflikt ein weiteres Mitglied der Steuergruppe mit keinem oder lediglich geringem Interessenkonflikt zur Seite gestellt wurde.

- Bei 2 Mandatsträgern wurden Patente festgestellt. Diese haben jedoch keinen thematischen Bezug zur Leitlinie und sind nicht kommerzialisiert. Es erfolgte daher kein Ausschluss von der Leitlinienarbeit.

- Ein Aktienbesitz lag bei keinem Mandatsträger vor. 


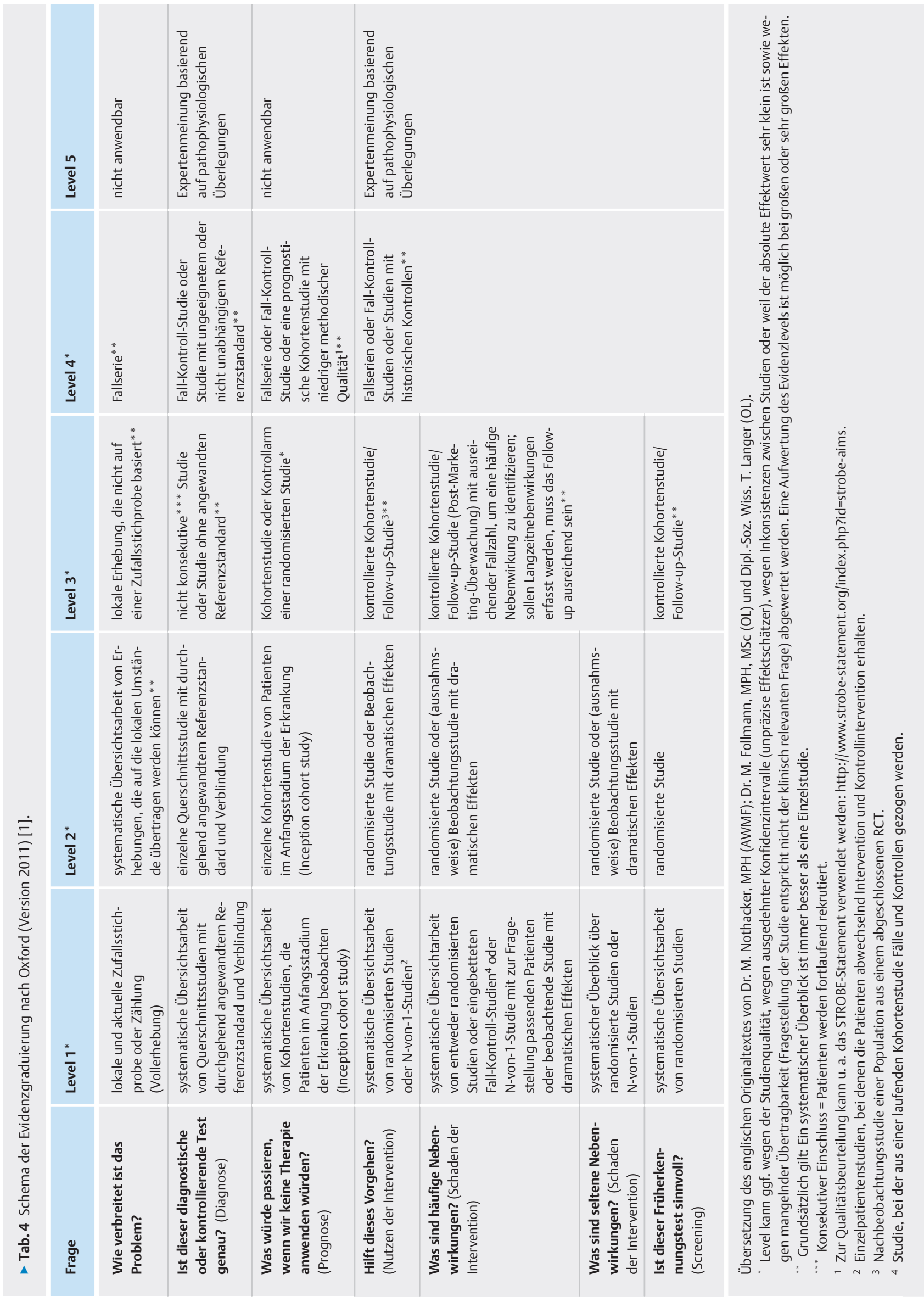


- Tab.5 Schema der Empfehlungsgraduierung.

\begin{tabular}{|l|l|l|}
\hline $\begin{array}{l}\text { Empfeh- } \\
\text { lungsgrad }\end{array}$ & Beschreibung & Ausdrucksweise \\
\hline $\mathbf{A}$ & starke Empfehlung & soll \\
\hline $\mathbf{B}$ & Empfehlung & sollte \\
\hline $\mathbf{0}$ & Empfehlung offen & kann \\
\hline
\end{tabular}

Umgang mit indirekten sekundären Interessenkonflikten:

- Mitgliedschaften in Fachgesellschaften und Beteiligung an Fortbildungen und Ausbildungsinstituten wurden nicht als Interessenkonflikt für diese Leitlinie bewertet, da diese ein essenzieller Teil der wissenschaftlichen und klinischen Arbeit sind.

- Ebenso wurde der Schwerpunkt der wissenschaftlichen und klinischen Tätigkeit in diesem Feld erwartet, um eine wissenschaftliche und praktikable Leitlinie zu erstellen.

- Eine persönliche Beziehung (Partner oder Verwandter 1. Grades) zu einem Vertretungsberechtigten eines Unternehmens der Gesundheitswirtschaft lag bei keinem Mandatsträger vor.
D Tab. 6 Konsensstärke.

\begin{tabular}{|l|l|}
\hline Konsensstärke & prozentuale Zustimmung \\
\hline starker Konsens & $>95 \%$ der Stimmberechtigten \\
\hline Konsens & $>75-95 \%$ der Stimmberechtigten \\
\hline $\begin{array}{l}\text { mehrheitliche } \\
\text { Zustimmung }\end{array}$ & $50-75 \%$ der Stimmberechtigten \\
\hline Dissens & $<50 \%$ der Stimmberechtigten \\
\hline
\end{tabular}

Der Vorschlag der Steuergruppe zum Management wurde zu Beginn der Konsenskonferenz diskutiert und umgesetzt.

Als protektive Faktoren gegen eine Verzerrung durch Interessenkonflikte können die systematische Evidenzaufbereitung, die pluralistische Zusammensetzung der Leitliniengruppe, die neutrale Moderation, die Diskussion der Bewertung der Interessen und des Umgangs mit Interessenkonflikten zu Beginn der Konsenskonferenz sowie die öffentliche Konsultation gewertet werden.

An dieser Stelle möchten wir allen Mitarbeitern für ihre ausschließlich ehrenamtliche Mitarbeit an dem Projekt danken.

\section{Diagnostik und Therapie des hepatozellulären Karzinoms}

\subsection{Risikofaktoren, Prävention und Früherkennung}

\subsubsection{Risikofaktoren und Früherkennung}

\begin{tabular}{|c|c|c|c|c|}
\hline Nr. & Empfehlung/Statement & EG & LoE & Quellen \\
\hline 3.1.a & Patienten mit einer Leberzirrhose haben ein erhöhtes Risiko für die Entwicklung eines HCC. & & 1 & $\begin{array}{l}\text { AASLD } \\
{[3]}\end{array}$ \\
\hline 3.1.b & Diesen Patienten sollte eine regelmäßige Früherkennung angeboten werden. & EK & & \\
\hline 3.2. & $\begin{array}{l}\text { Patienten mit einer chronischen Hepatitis-B-Virusinfektion haben ein erhöhtes Risiko für die } \\
\text { Entwicklung eines HCC. Diesen Patienten sollte ab einem PAGE-B-Score von } 10 \text { eine regelmäßige } \\
\text { Früherkennung angeboten werden. }\end{array}$ & B & 2 & $\begin{array}{l}\text { de novo } \\
{[4,5]}\end{array}$ \\
\hline 3.3. & $\begin{array}{l}\text { Patienten mit chronischer HCV-Infektion oder nichtalkoholischer Steatohepatitis haben bereits } \\
\text { bei einer fortgeschrittenen Leberfibrose ein erhöhtes Risiko für die Entwicklung eines HCC. Diesen } \\
\text { Patienten sollte eine regelmäßige Früherkennung angeboten werden. }\end{array}$ & EK & & \\
\hline 3.4. & $\begin{array}{l}\text { Patienten mit einer fortgeschrittenen Leberfibrose, unabhängig von der Ursache der } \\
\text { Lebererkrankung, kann die Teilnahme an einer HCC-Früherkennung angeboten werden. }\end{array}$ & EK & & \\
\hline
\end{tabular}




\subsubsection{Prophylaktische und therapeutische Maßnahmen zur Reduktion des HCC-Risikos}

\begin{tabular}{|c|c|c|c|c|}
\hline Nr. & Empfehlung/Statement & EG & LoE & Quellen \\
\hline 3.5. & $\begin{array}{l}\text { Entsprechend den Empfehlungen der Ständigen Impfkommission am Robert-Koch-Institut (STIKO) } \\
\text { sollen gegen das Hepatitis-B-Virus geimpft werden: } \\
\text { - alle Säuglinge ab dem vollendeten 2. Lebensmonat } \\
\text { - alle Neugeborenen HBsAg-positiver Mütter oder von Müttern mit unbekanntem HBsAg-Status } \\
\text { innerhalb von } 12 \text { Stunden } \\
\text { - alle noch nicht geimpften Kinder und Jugendlichen bis zum vollendeten 17. Lebensjahr, } \\
\text { möglichst vor Beginn der Pubertät } \\
\text { - alle in der Indikationsliste der STIKO aufgeführten Personen mit erhöhtem } \\
\text { Hepatitis-B-Risiko }\end{array}$ & EK & & \\
\hline 3.6. & $\begin{array}{l}\text { Die Ursachen einer chronischen Lebererkrankung sollen, soweit möglich, behandelt werden, } \\
\text { um eine Progression einschließlich der Entwicklung eines HCC zu verhindern. }\end{array}$ & EK & & \\
\hline 3.7. & $\begin{array}{l}\text { Patienten mit chronischer Hepatitis-Virusinfektion sollen gemäß den aktuellen Leitlinien betreut } \\
\text { bzw. antiviral behandelt werden, um eine Progression einschließlich der Entwicklung eines HCC } \\
\text { zu verhindern. }\end{array}$ & EK & & \\
\hline 3.8. & Patienten mit chronischen Lebererkrankungen sollte der Konsum von Kaffee empfohlen werden. & B & 2 & $\begin{array}{l}\text { EASL } \\
{[6-10]}\end{array}$ \\
\hline 3.9. & $\begin{array}{l}\text { Bei Patienten mit chronischer Lebererkrankung und nicht insulinabhängigem Diabetes mellitus } \\
\text { sollte eine Behandlung mit Metformin geprüft werden, um das HCC-Risiko zu senken. }\end{array}$ & EK & & \\
\hline 3.10. & Patienten mit chronischen Lebererkrankungen sollen keinen Alkohol trinken. & EK & & \\
\hline 3.11. & $\begin{array}{l}\text { Patienten mit chronischer Hepatitis-B-Virusinfektion und HCC sollte eine antivirale Therapie ange- } \\
\text { boten werden. }\end{array}$ & EK & & \\
\hline 3.12 . & $\begin{array}{l}\text { Patienten mit chronischer Hepatitis-C-Virusinfektion und HCC, bei denen eine kurativ intendierte } \\
\text { Tumorbehandlung durchgeführt wird, soll eine DAAD-Behandlung angeboten werden. }\end{array}$ & EK & & \\
\hline
\end{tabular}

\subsubsection{Vorsorgeuntersuchung}

\begin{tabular}{|c|c|c|c|c|}
\hline Nr. & Empfehlung/Statement & EG & LoE & Quellen \\
\hline 3.13. & $\begin{array}{l}\text { Patienten mit Leberzirrhose im Stadium Child-Pugh A und B soll die Teilnahme an einer } \\
\text { HCC-Früherkennung angeboten werden. }\end{array}$ & A & 2 & $\begin{array}{l}\text { de novo } \\
{[11]}\end{array}$ \\
\hline 3.14 & $\begin{array}{l}\text { Patienten mit Leberzirrhose, die zur Lebertransplantation gelistet sind, soll die Teilnahme an einer } \\
\text { HCC-Früherkennung angeboten werden. }\end{array}$ & EK & & \\
\hline 3.15. & $\begin{array}{l}\text { Im Rahmen der HCC-Früherkennung soll alle } 6 \text { Monate eine Ultraschalluntersuchung der Leber } \\
\text { durchgeführt werden. }\end{array}$ & A & 2 & $\begin{array}{l}\text { EASL } \\
{[6,11-13]}\end{array}$ \\
\hline 3.16. & Die Früherkennung kann durch eine AFP-Bestimmung ergänzt werden. & 0 & 1 & $\begin{array}{l}\text { de novo } \\
{[14]}\end{array}$ \\
\hline 3.17. & $\begin{array}{l}\text { Bei Patienten mit chronischer Lebererkrankung sollte das Fibrosestadium wiederholt erhoben } \\
\text { werden, um das HCC-Risiko besser einzuschätzen. }\end{array}$ & B & 3 & $\begin{array}{l}\text { de novo } \\
{[15,16]}\end{array}$ \\
\hline
\end{tabular}




\subsection{Histopathologische und molekulare Diagnostik}

\begin{tabular}{|c|c|c|c|c|}
\hline Nr. & Empfehlung/Statement & EG & LoE & Quellen \\
\hline 3.18. & $\begin{array}{l}\text { In der palliativen Situation und bei unklarem Kontrastmittelverhalten in } 2 \text { unabhängigen } \\
\text { Bildgebungen in der kurativen Situation sollte ein bioptisches Verfahren eingesetzt werden. }\end{array}$ & EK & & \\
\hline 3.19. & $\begin{array}{l}\text { Die Typisierung des HCC soll sich nach der aktuellen WHO-Klassifikation richten. } \\
\text { Hierbei sollten zum einen Sonderformen (z. B. fibrolamelläres HCC und mischdifferenzierte } \\
\text { Tumoren (kombiniertes HCC/iCCA)) und wenn möglich auch das frühe HCC vom progredienten } \\
\text { HCC und prämalignen Läsionen unterschieden werden. } \\
\text { Es sollte eine sichere Unterscheidung von Sonderformen des intrahepatischen } \\
\text { Cholangiokarzinoms, Lebermetastasen und auch benignen Lebertumoren erfolgen. }\end{array}$ & EK & & \\
\hline 3.20 . & $\begin{array}{l}\text { Die Bearbeitung und Befundung eines Resektats oder Explantats sollen die Ausdehnung } \\
\text { des Tumors (Staging) gemäß der aktuellen TNM-Klassifikation, seinen Typ (Typing) und Differen- } \\
\text { zierungsgrad (Grading) und den Status des Resektatrands (R-Klassifikation) sowie den Status } \\
\text { der nichttumorösen Leber ermitteln. }\end{array}$ & EK & & \\
\hline 3.21. & $\begin{array}{l}\text { Wenn die Diagnose eines HCC nicht mithilfe der konventionellen Histologie gestellt werden kann, } \\
\text { sollen in Abhängigkeit vom histopathologischen Erscheinungsbild weitere, insbesondere immun- } \\
\text { histologische und/oder molekularpathologische Untersuchungen zur Sicherung der Diagnose } \\
\text { eingesetzt werden. }\end{array}$ & EK & & \\
\hline 3.22. & $\begin{array}{l}\text { Molekularpathologische Untersuchungen können zur Unterstützung der Tumortypisierung und } \\
\text { Dignitätsbestimmung von hepatozellulären Tumoren eingesetzt werden. Prädiktive molekulare } \\
\text { Analysen sind in der regulären Diagnostik noch nicht erforderlich, können aber zur erweiterten } \\
\text { Therapieplanung eingesetzt werden. }\end{array}$ & EK & & \\
\hline
\end{tabular}

\subsection{Bildgebende Diagnostik}

\begin{tabular}{|c|c|c|c|c|}
\hline Nr. & Empfehlung/Statement & EG & LoE & Quellen \\
\hline 3.23. & Die Sonografie kann zur initialen Einschätzung im Risikokollektiv verwendet werden. & \multicolumn{3}{|l|}{ EK } \\
\hline 3.24 . & $\begin{array}{l}\text { Das HCC in der zirrhotischen Leber soll bildgebend anhand seiner typischen Kontrastmitteldynamik } \\
\text { mit arterieller Hypervaskularisation und Auswaschen in der portalvenösen und venösen Phase } \\
\text { im kontrastverstärkten MRT diagnostiziert werden. }\end{array}$ & A & 2 & $\begin{array}{l}\text { de novo } \\
{[17-19]}\end{array}$ \\
\hline 3.25 . & $\begin{array}{l}\text { Ein triphasisches CT und/oder CEUS sollten bei unklarem MRT-Befund oder Kontraindikationen } \\
\text { für das MRT zur weiteren Diagnostik herangezogen werden. }\end{array}$ & \multicolumn{3}{|l|}{ EK } \\
\hline 3.26 . & $\begin{array}{l}\text { Das Staging eines HCC soll ein kontrastverstärktes CT des Thorax und des Abdomens beinhalten. } \\
\text { Falls das kontrastverstärkte MRT das gesamte Abdomen diagnostisch abdeckt, soll lediglich } \\
\text { ein nativer CT-Thorax ergänzt werden. }\end{array}$ & \multicolumn{3}{|l|}{ EK } \\
\hline 3.27. & $\begin{array}{l}\text { Unklare Leberherde }<1 \mathrm{~cm} \text { sollten in 3-Monats-Intervallen mittels des bestgeeigneten kontrast- } \\
\text { mittelverstärkten Schnittbildverfahrens kontrolliert werden. }\end{array}$ & B & 4 & \multirow[t]{2}{*}{$\begin{array}{l}\text { de novo } \\
{[20]}\end{array}$} \\
\hline 3.28 . & $\begin{array}{l}\text { Bei Leberherden } \geq 1 \mathrm{~cm} \text { und untypischem Kontrastmittelverhalten sollte bei kurativer } \\
\text { Behandlungsindikation eine zweite kontrastmittelverstärkte Bildgebung mittels CT und/oder CEUS } \\
\text { erfolgen. }\end{array}$ & $\mathrm{B}$ & 4 & \\
\hline 3.29 . & $\begin{array}{l}\text { Bei Leberherden } \geq 1 \mathrm{~cm} \text { und untypischem Kontrastmittelverhalten, bei denen kein kurativer Ansatz } \\
\text { besteht, soll bei Indikation für eine tumorgerichtete Therapie eine Biopsie erfolgen. }\end{array}$ & EK & & \\
\hline
\end{tabular}

\subsubsection{Diagnostikalgorithmus}

Malignitätsverdächtige Leberrundherde sollen in einer therapierelevanten Konstellation unmittelbar abgeklärt werden (siehe auch „Diagnosealgorithmus eines Patienten mit einer HCC-verdächtigen Raumforderung“) ( $\triangleright$ Abb. 1: Diagnostikalgorithmus). 


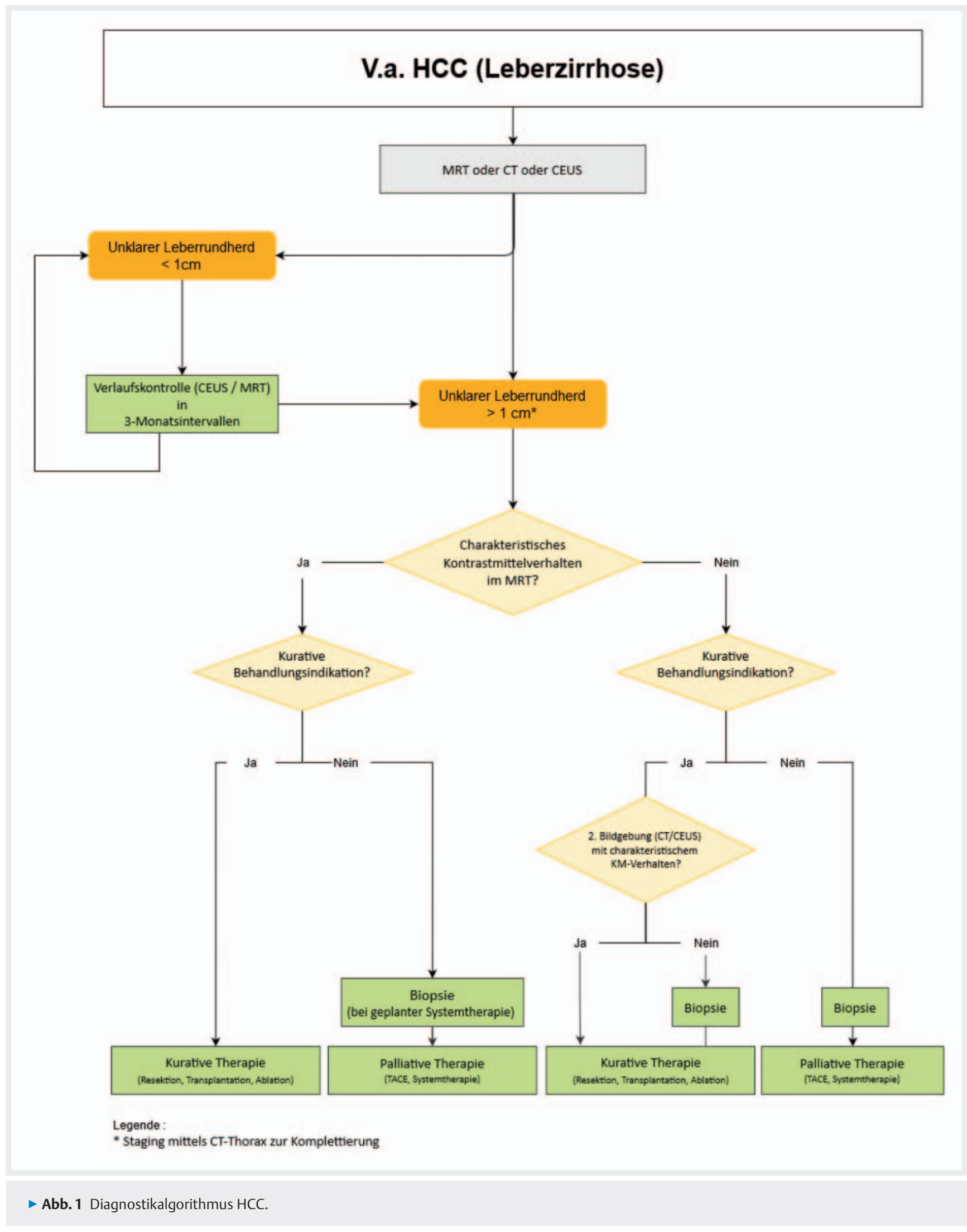


3.3.2. Staging-Systeme (Clip, TNM, Okuda, BCLC etc.)

\begin{tabular}{|c|c|c|c|c|}
\hline Nr. & Empfehlung/Statement & EG & LoE & Quellen \\
\hline 3.30 . & $\begin{array}{l}\text { Für die klinische Entscheidungsfindung bei HCC sollen die Tumorlast, die Leberfunktion } \\
\text { und der Leistungsstatus berücksichtigt werden. }\end{array}$ & A & 2 & $\begin{array}{l}\text { EASL } \\
{[6,21-29]}\end{array}$ \\
\hline 3.31. & $\begin{array}{l}\text { Zur Stratifizierung vor Operationen/Transplantationen soll ergänzend die } \\
\text { TNM-Klassifikation verwendet werden. }\end{array}$ & EK & & \\
\hline 3.32 . & $\begin{array}{l}\text { Bezüglich der bildmorphologischen Tumoraspekte sollen bildgebende Analyseverfahren } \\
\text { verwendet werden, die die Vaskularität berücksichtigen. }\end{array}$ & EK & & \\
\hline
\end{tabular}

\subsection{Operative und interventionelle Therapieverfahren}

\author{
Nr. \\ Empfehlung/Statement \\ 3.33. \\ Patienten mit einem hepatozellulären Karzinom sollen in einer interdisziplinären Tumorkonferenz \\ vorgestellt werden.
}

EG

LoE

EK, Klug entscheiden

\subsubsection{Operative Therapieverfahren}

\subsubsection{Lebertransplantation}

\begin{tabular}{|c|c|c|c|c|}
\hline Nr. & Empfehlung/Statement & EG & LoE & Quellen \\
\hline 3.34. & $\begin{array}{l}\text { Patienten mit einem kurativ behandelbaren hepatozellulären Karzinom sollten in einem } \\
\text { Lebertransplantationszentrum vorgestellt werden. }\end{array}$ & \multicolumn{3}{|c|}{ EK, Klug entscheiden } \\
\hline 3.35. & $\begin{array}{l}\text { Geeignete Patienten mit Leberzirrhose und einem irresektablen HCC innerhalb der Mailand- } \\
\text { Kriterien (BCLC A) sollen für eine Lebertransplantation evaluiert werden. }\end{array}$ & \multicolumn{3}{|l|}{ EK } \\
\hline 3.36. & $\begin{array}{l}\text { Auch bei formal resektablen oder grenzwertig resektablen HCC-Befunden in Zirrhose } \\
\text { kann innerhalb der Mailand-Kriterien eine Indikation zur Transplantation bestehen. }\end{array}$ & \multicolumn{3}{|l|}{ EK } \\
\hline 3.37. & $\begin{array}{l}\text { Bei extrahepatischen Tumormanifestationen und/oder einer makrovaskulären Invasion } \\
\text { der Lebergefäße soll eine Lebertransplantation nicht durchgeführt werden. }\end{array}$ & \multicolumn{3}{|l|}{ EK } \\
\hline 3.38 & $\begin{array}{l}\text { Bei einem AFP-Wert > } 1000 \mathrm{ng} / \mathrm{ml} \text { sollte keine Indikation zur Transplantation ohne neoadjuvante } \\
\text { Therapie gestellt werden. }\end{array}$ & \multicolumn{3}{|l|}{ EK } \\
\hline 3.39. & $\begin{array}{l}\text { Bei AFP-Anstieg auf }>1000 \mathrm{ng} / \mathrm{ml} \text { unter Bridging-Therapie sollte die Transplantation } \\
\text { nicht durchgeführt werden. }\end{array}$ & \multicolumn{3}{|l|}{ EK } \\
\hline 3.40 & $\begin{array}{l}\text { Das chronologische Alter stellt per se keine Kontraindikation dar. Wenn Patienten älter als } 65 \text { Jahre } \\
\text { sind, sollte die Indikation zur Lebertransplantation allerdings besonders sorgfältig geprüft werden. }\end{array}$ & \multicolumn{3}{|l|}{ EK } \\
\hline
\end{tabular}

- Tab.7 Übersicht der gängigsten Selektionskriterien zur Lebertransplantation.

\begin{tabular}{|c|c|c|c|}
\hline & solitärer Knoten & mehrere Tumorknoten & $\begin{array}{l}\text { 5-Jahres-Überlebensrate nach } \\
\text { Lebertransplantation }\end{array}$ \\
\hline & \multicolumn{3}{|c|}{$\begin{array}{l}\text { keine extrahepatischen Manifestationen (cNOM0) } \\
\text { keine makrovaskuläre Gefäßinvasion (cV0) }\end{array}$} \\
\hline Mailand-Kriterien & $\begin{array}{l}\leq 5 \mathrm{~cm} \\
(B \ddot{K} K \geq 1 \mathrm{~cm} \text { und } \leq 5 \mathrm{~cm})\end{array}$ & $\begin{array}{l}\text { max. } 3 \text { Herde } \leq 3 \mathrm{~cm} \\
(B \ddot{K K} \geq 1 \mathrm{~cm} \text { und } \leq 3 \mathrm{~cm} \text { ) }\end{array}$ & $>70 \%$ \\
\hline Up-to-7-Kriterien & $\leq 7 \mathrm{~cm}$ & $\begin{array}{l}\text { Summe aus Durchmesser des größten Tumors } \\
(\mathrm{cm}) \text { und Anzahl der Tumorknoten } \leq 7\end{array}$ & $>70 \%$ \\
\hline UCSF-Kriterien & $\leq 6,5 \mathrm{~cm}$ & $\begin{array}{l}\text { max. } 3 \text { Herde } \leq 4,5 \mathrm{~cm} \text { und eine maximale Summe } \\
\text { der addierten Tumordurchmesser } \leq 8 \mathrm{~cm}\end{array}$ & $>70 \%$ \\
\hline
\end{tabular}


Tab. 8 Definitionen.

\begin{tabular}{|l|l|}
\hline Begriff & Definition \\
\hline neoadjuvante Therapie & $\begin{array}{l}\text { Behandlung, die vor einer operativen Maßnahme (z. B. Resektion oder Lebertransplantation) erfolgt, mit dem Ziel einer } \\
\text { Verkleinerung der Tumormasse. }\end{array}$ \\
\hline Bridging & Lokoregionäre Therapie oder Resektion eines HCC innerhalb der Mailand-Kriterien auf der Warteliste. \\
\hline Downstaging & $\begin{array}{l}\text { Vorbehandlung eines HCC außerhalb der Mailand-Kriterien mit dem Ziel der Tumorverkleinerung bis Zu definierten } \\
\text { Selektionskriterien (in der Regel: bis die Mailand-Kriterien erreicht sind). }\end{array}$ \\
\hline
\end{tabular}

\subsection{Bridging-Therapie}

\begin{tabular}{|c|c|c|c|c|}
\hline Nr. & Empfehlung/Statement & EG & LoE & Quellen \\
\hline 3.41 & $\begin{array}{l}\text { Vor Beginn einer Bridging-Therapie soll eine Kontaktaufnahme mit einem Transplantationszentrum } \\
\text { erfolgen. }\end{array}$ & \multicolumn{3}{|l|}{ EK } \\
\hline 3.42. & $\begin{array}{l}\text { Patienten mit HCC (BCLC A) innerhalb der Mailand-Kriterien sollen eine Bridging-Therapie erhalten, } \\
\text { sofern es die Leberfunktion zulässt. }\end{array}$ & A & 1 & \multirow[t]{2}{*}{$\begin{array}{l}\text { de novo } \\
{[30-32]}\end{array}$} \\
\hline 3.43. & $\begin{array}{l}\text { Für das Bridging sollen Lokalablation, Resektion oder transarterielle Verfahren (TACE, TARE) } \\
\text { eingesetzt werden. }\end{array}$ & A & 1 & \\
\hline 3.44 & $\begin{array}{l}\text { Eine Hochpräzisionsradiotherapie (Stereotactic Body Radiotherapy, SBRT) kann als Bridging- } \\
\text { Verfahren bis zur Lebertransplantation eingesetzt werden, wenn die weiteren Bridging-Verfahren } \\
\text { nicht zum Einsatz kommen können. }\end{array}$ & EK & & \\
\hline
\end{tabular}

\subsubsection{Downstaging}

\begin{tabular}{|c|c|c|c|c|}
\hline Nr. & Empfehlung/Statement & EG & LoE & Quellen \\
\hline 3.45. & $\begin{array}{l}\text { Bei geeigneten Patienten mit einem HCC außerhalb der Mailand-Kriterien und innerhalb } \\
\text { der UCSF-Kriterien kann eine Lebertransplantation erfolgen, insbesondere dann, wenn ein } \\
\text { Downstaging bis innerhalb der Mailand-Kriterien gelingt. }\end{array}$ & 0 & 1 & $\begin{array}{l}\text { de novo } \\
{[31,33,34]}\end{array}$ \\
\hline 3.46. & $\begin{array}{l}\text { Außerhalb der UCSF-Kriterien kann bei Tumoren ohne extrahepatische Manifestation und ohne } \\
\text { Makroinvasion von Gefäßen nach Ansprechen auf eine neoadjuvante Therapie eine Evaluation } \\
\text { zur Lebertransplantation erwogen werden. }\end{array}$ & EK & & \\
\hline 3.47 & $\begin{array}{l}\text { Für das Downstaging sollen entweder eine Lokalablation, eine Resektion oder ein transarterielles } \\
\text { Verfahren (TACE, TARE) eingesetzt werden. }\end{array}$ & EK & & \\
\hline
\end{tabular}

\subsection{Nachsorge nach Lebertransplantation}

\begin{tabular}{|l|l|l|l|}
\hline Nr. & Empfehlung/Statement & Quellen \\
\hline 3.48. & $\begin{array}{l}\text { Die Tumornachsorge sollte nach den allgemeinen Empfehlungen der HCC-Nachsorge } \\
\text { (siehe Kapitel Nachsorge } 0) \text { erfolgen. }\end{array}$ & LK \\
\hline 3.49. & $\begin{array}{l}\text { Außerhalb von Studien sollen Patienten mit HCC nach Lebertransplantation nicht adjuvant } \\
\text { behandelt werden. }\end{array}$ & EK \\
\hline
\end{tabular}




\subsubsection{Resektion}

\subsection{Resektion beim hepatozellulären Karzinom ohne Leberzirrhose}

\begin{tabular}{|l|l|l|l|}
\hline Nr. & Empfehlung/Statement & EG \\
\hline 3.50. & $\begin{array}{l}\text { Eine Resektion eines HCC ohne Leberzirrhose soll erfolgen, wenn eine komplette Resektion } \\
\text { (R0-Resektion) möglich ist. }\end{array}$ & EK \\
\hline 3.51. & $\begin{array}{l}\text { Nach Resektion eines HCC ohne Leberzirrhose sollte eine regelmäßige Nachsorge über } 5 \text { Jahre } \\
\text { erfolgen. }\end{array}$ & EK \\
\hline
\end{tabular}

\subsection{Resektion beim hepatozellulären Karzinom mit Leberzirrhose}

\begin{tabular}{|c|c|c|c|c|}
\hline Nr. & Empfehlung/Statement & EG & LoE & Quellen \\
\hline 3.52. & $\begin{array}{l}\text { Bei einem singulären HCC-Knoten in Leberzirrhose mit funktioneller Resektabilität sollte eine } \\
\text { Leberresektion erfolgen. Dies trifft insbesondere für HCC-Knoten }>3 \mathrm{~cm} \text { zu. }\end{array}$ & \multicolumn{3}{|l|}{ EK } \\
\hline 3.53. & $\begin{array}{l}\text { Bei multiplen HCC-Knoten in Leberzirrhose innerhalb der Mailand-Kriterien kann eine Leberresek- } \\
\text { tion durchgeführt werden. }\end{array}$ & \multicolumn{3}{|l|}{ EK } \\
\hline 3.54. & $\begin{array}{l}\text { Die Leberresektion kann offen oder minimalinvasiv durchgeführt werden. Besonders bei zukünftigen } \\
\text { Lebertransplantationspatienten sollte eine minimalinvasive Leberresektion durchgeführt werden. }\end{array}$ & \multicolumn{3}{|l|}{ EK } \\
\hline 3.55. & Eine neoadjuvante oder adjuvante Therapie bei Resektion soll nur im Rahmen von Studien erfolgen. & A & 2 & $\begin{array}{l}\text { EASL } \\
{[6,35-39]}\end{array}$ \\
\hline 3.56. & Nach Leberresektion beim HCC in Zirrhose soll eine regelmäßige Nachsorge erfolgen. & A & 2 & $\begin{array}{l}\text { EASL } \\
{[6,40-45]}\end{array}$ \\
\hline
\end{tabular}

\subsubsection{Interventionelle Therapieverfahren}

\subsubsection{Perkutane Ablation}

\begin{tabular}{|c|c|c|c|c|}
\hline Nr. & Empfehlung/Statement & EG & LoE & Quellen \\
\hline 3.57. & Bei Patienten mit HCC bis $3 \mathrm{~cm}$ sind die Resektion und die Ablation äquivalente Verfahren. & \multicolumn{3}{|l|}{ EK } \\
\hline 3.58. & $\begin{array}{l}\text { Patienten mit HCC kleiner } 3 \mathrm{~cm} \text { in für die Resektion ungünstiger Lokalisation oder mit einge- } \\
\text { schränkter Leberfunktion soll primär eine Thermoablation des Tumors angeboten werden. }\end{array}$ & A & 1 & $\begin{array}{l}\text { EASL } \\
{[6,46,47]}\end{array}$ \\
\hline 3.59. & $\begin{array}{l}\text { Die perkutane Ablation des HCC soll mittels Radiofrequenzablation (RFA) oder Mikrowellenablation } \\
\text { (MWA) durchgeführt werden }\end{array}$ & \multicolumn{3}{|l|}{ EK } \\
\hline 3.60. & $\begin{array}{l}\text { Bei Patienten mit erhaltener Leberfunktion und gering- oder mäßiggradiger portaler Hypertension } \\
\text { soll, bei einem HCC-Herd }>3 \mathrm{~cm} \text { und }<5 \mathrm{~cm} \text {, vor einer Thermoablation chemoembolisiert werden. }\end{array}$ & A & 2 & $\begin{array}{l}\text { de novo } \\
{[48-50]}\end{array}$ \\
\hline
\end{tabular}

\subsubsection{Transarterielle Chemoembolisation (TACE)}

\begin{tabular}{|c|c|c|c|c|}
\hline Nr. & Empfehlung/Statement & EG & LoE & Quellen \\
\hline 3.61. & $\begin{array}{l}\text { Die TACE soll Patienten mit HCC im intermediären Stadium angeboten werden, wenn keine } \\
\text { kurativen Therapieoptionen vorliegen. }\end{array}$ & A & 2 & $\begin{array}{l}\text { de novo } \\
{[51,52]}\end{array}$ \\
\hline 3.62. & Die TACE setzt eine erhaltene Leberfunktion voraus. & & 2 & $\begin{array}{l}\text { de novo } \\
{[53]}\end{array}$ \\
\hline 3.63. & $\begin{array}{l}\text { Die TACE soll dem Vaskularisationsmuster der Tumorherde angepasst werden und so selektiv } \\
\text { wie möglich erfolgen. }\end{array}$ & EK & & \\
\hline 3.64. & Konventionelle TACE und Drug-eluting-TACE können als gleichwertige Verfahren angesehen werden. & & 2 & $\begin{array}{l}\text { de novo } \\
{[53,54]}\end{array}$ \\
\hline
\end{tabular}




\begin{tabular}{|l|l|l|}
\hline Nr. & Empfehlung/Statement & Quellen \\
\hline 3.65. & $\begin{array}{l}\text { Die TACE sollte mehrfach durchgeführt werden, solange ein Ansprechen hierauf nachweisbar ist } \\
\text { (CR, PR nach mRECIST) und behandelbare hypervaskularisierte Tumoranteile verbleiben. }\end{array}$ & EK \\
\hline 3.66. & $\begin{array}{l}\text { Die Indikation zur Fortführung der TACE soll nach } 2 \text { Behandlungszyklen im Tumorboard überprüft } \\
\text { werden. }\end{array}$ & EK \\
\hline
\end{tabular}

\subsubsection{Transarterielle Radioembolisation (TARE)}

\begin{tabular}{|l|l|l|l|l|l|l|l|l|}
\hline Nr. & Empfehlung/Statement & Luellen & LG \\
\hline 3.67. & $\begin{array}{l}\text { Die TARE kann nach Beschluss des Tumorboards bei Patienten mit erhaltener Leberfunktion } \\
\text { im intermediären HCC-Stadium anstelle einer TACE eingesetzt werden. }\end{array}$ & 2 \\
\hline
\end{tabular}

\subsubsection{Stereotaxie}

\begin{tabular}{|l|l|l|}
\hline Nr. & Empfehlung/Statement & EG \\
\hline 3.68. & $\begin{array}{l}\text { Eine Hochpräzisionsradiotherapie (Stereotactic Body Radiotherapy, SBRT) kann in Betracht } \\
\text { gezogen werden, wenn andere lokale Therapieverfahren nicht möglich sind (z. B. hohe Wahr- } \\
\text { scheinlichkeit für ein Therapieversagen, eingeschränkte Leberfunktion, technische Hindernisse). }\end{array}$ \\
\hline
\end{tabular}

3.4.4. Nachsorge und Erfolgskontrolle nach interventioneller Therapie

\begin{tabular}{|l|l|l|l|}
\hline Nr. & Empfehlung/Statement & EG & LoE \\
\hline 3.69 & $\begin{array}{l}\text { Erfolgskontrollen nach lokaler Therapie sollten mittels biphasischer CECT oder dynamischer MRT in } \\
\text { einem Intervall von 4-12 Wochen nach Ablation/Resektion bzw. nach jedem TACE-Zyklus erfolgen. }\end{array}$ & EK \\
\hline 3.70. & $\begin{array}{l}\text { Die Verlaufskontrolle nach erfolgreicher lokaler Therapie soll im ersten Jahr alle 3 Monate und } \\
\text { im zweiten Jahr alle 3-6 Monate mittels biphasischer CECT oder dynamischer MRT stattfinden. }\end{array}$ & EK \\
\hline 3.71. & $\begin{array}{l}\text { Nach Abschluss der Nachsorge sollen die Patienten wieder in das Früherkennungsprogramm } \\
\text { mit Ultraschall alle 6 Monate eingeschlossen werden. }\end{array}$ & EK & EK \\
\hline 3.72. & Die Remissionsbeurteilung nach Ablation/TACE/TARE soll nach mRECIST/EASL erfolgen. & \\
\hline
\end{tabular}




\subsubsection{Therapiealgorithmus}

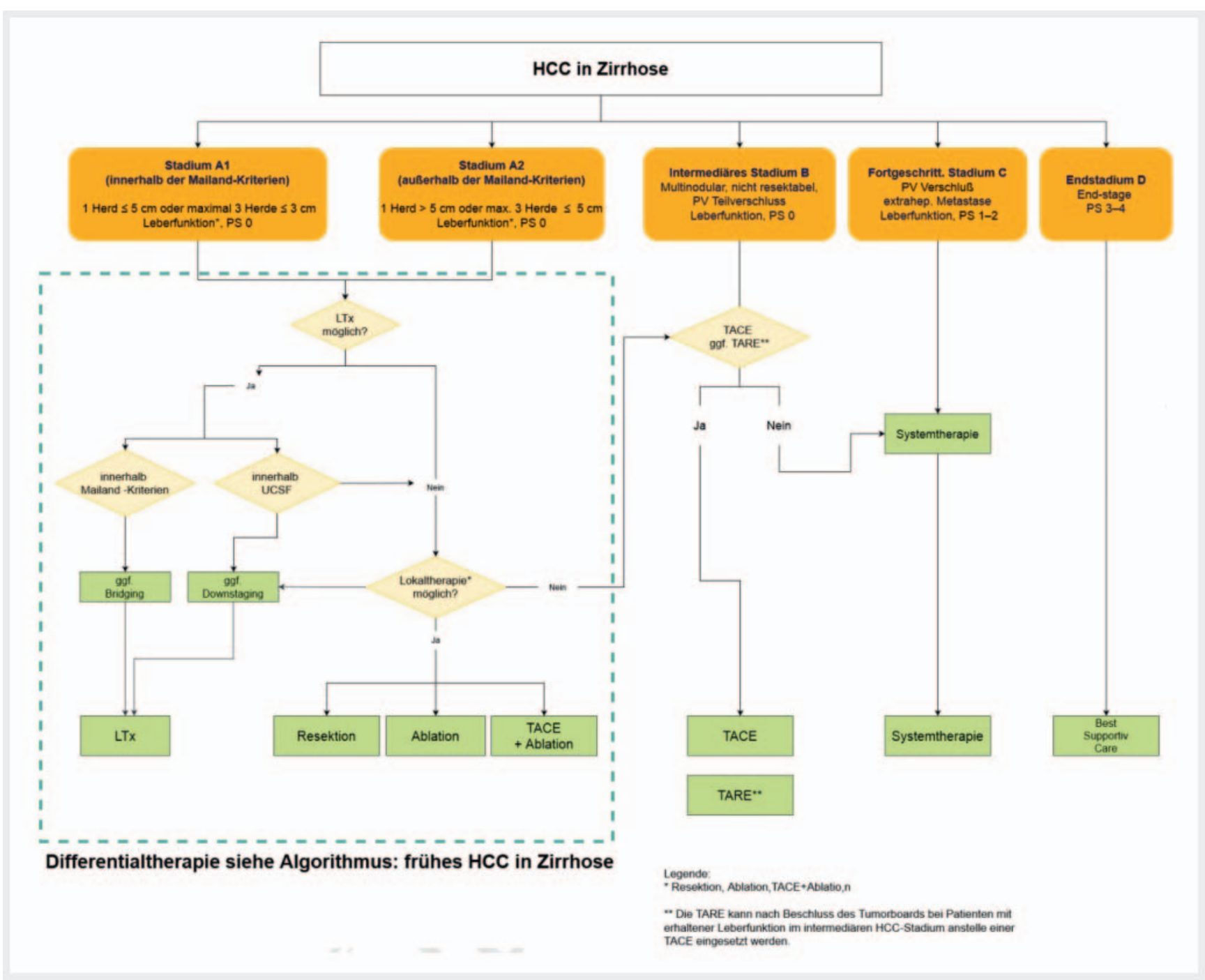

- Abb. 2 Therapiealgorithmus HCC in Zirrhose. 


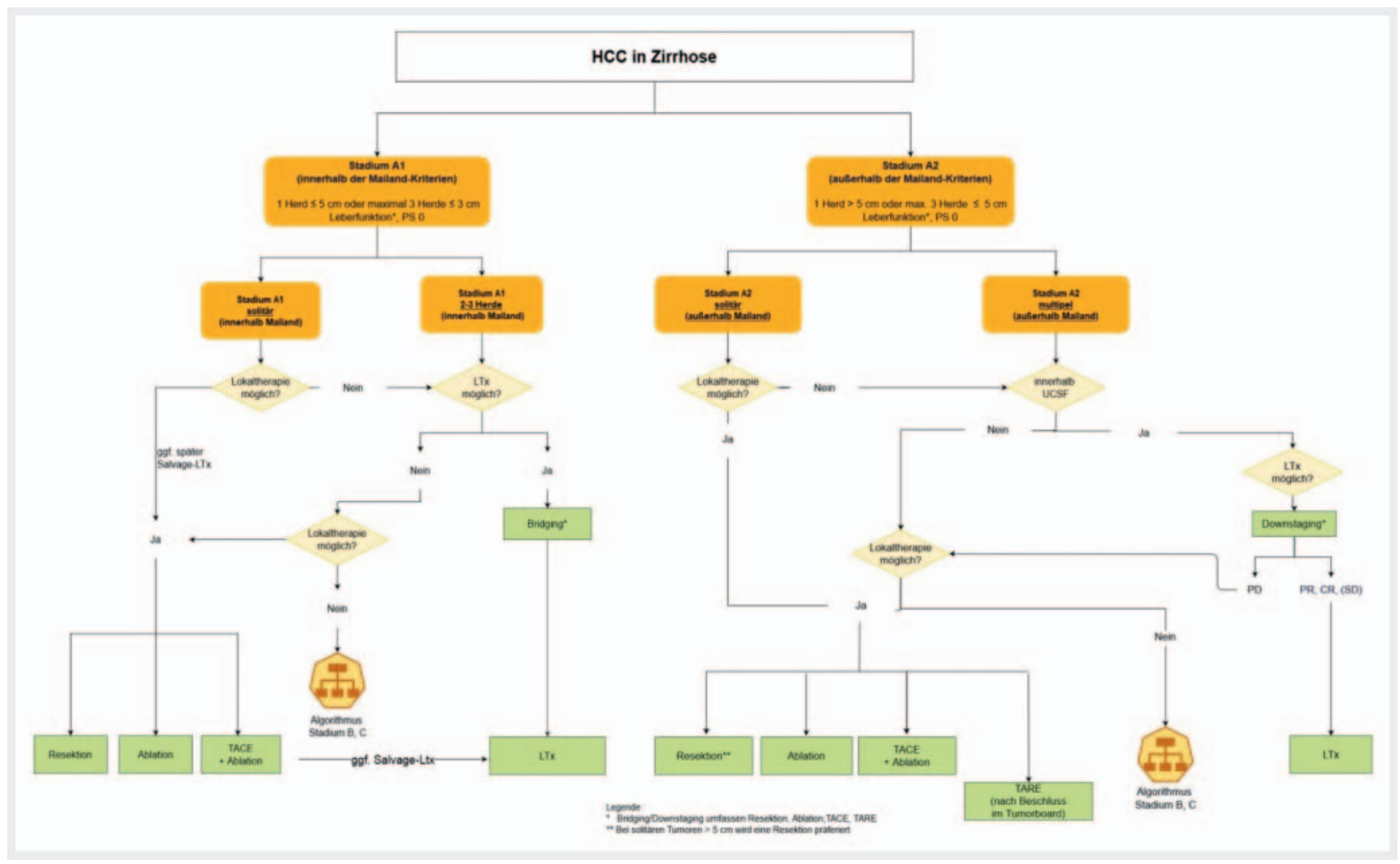

- Abb. 3 Therapiealgorithmus des HCC in Zirrhose im Stadium A1 und A2.

\subsection{Systemtherapie}

3.5.1. Substanzen mit einem Wirksamkeitsnachweis in Phase-III-Studien für die Systemtherapie des HCC

\begin{tabular}{|c|c|c|c|c|}
\hline Nr. & Empfehlung/Statement & EG & LoE & Quellen \\
\hline 3.73. & $\begin{array}{l}\text { Für HCC-Patienten mit erhaltener Leberfunktion (im Child-Pugh-Stadium A), mit Fernmetastasen } \\
\text { und/oder einer Tumorlokalisation, die lokoregionär nicht kontrolliert oder reseziert werden kann, } \\
\text { liegen Phase-III-Studien mit Wirksamkeitsnachweis vor, für } \\
\text { I. die Kombinationstherapie mit den Antikörpern Atezolizumab gegen PD-L1 und Bevacizumab } \\
\text { gegen VEGF, } \\
\text { II. Tyrosinkinase-Inhibitoren mit Sorafenib und Lenvatinib, und nach einer Vortherapie mit Sorafenib } \\
\text { für Regorafenib und Cabozantinib sowie } \\
\text { III. den VEGF-R2-Antikörper Ramucirumab für Patienten nach Sorafenib und einem Alpha-Feto- } \\
\text { protein-Wert von } \geq 400 \mathrm{ng} / \mathrm{ml} \text {. }\end{array}$ & & $1-2$ & $\begin{array}{l}\text { de novo } \\
\text { [59-65] }\end{array}$ \\
\hline
\end{tabular}

\subsubsection{Medikamentöse Erstlinientherapie des HCC}

\begin{tabular}{|l|l|l|l|l|}
\hline Nr. & Empfehlung/Statement & Quellen \\
\hline 3.74. & $\begin{array}{l}\text { Eine Erstlinientherapie mit der Kombination Atezolizumab und Bevacizumab (A+B) soll angeboten } \\
\text { werden bei HCC-Patienten im Child-Pugh-Stadium A und BCLC B oder C, mit Fernmetastasen oder } \\
\text { einer Tumorlokalisation, die lokoregionär nicht kontrolliert oder reseziert werden kann. } \\
\text { Patienten mit einer Kontraindikation für A+B soll eine Erstlinientherapie mit einem der beiden } \\
\text { Tyrosinkinase-Inhibitoren Lenvatinib oder Sorafenib angeboten werden. }\end{array}$ \\
\hline
\end{tabular}


3.5.3. Medikamentöse Therapie nach Versagen, bei Unverträglichkeit oder bei Kontraindikationen der Erstlinientherapie des HCC

\begin{tabular}{|c|c|c|c|c|}
\hline Nr. & Empfehlung/Statement & EG & LoE & Quellen \\
\hline 3.75. & $\begin{array}{l}\text { Die Überlegenheit von Atezolizumab und Bevacizumab im Vergleich zu Sorafenib führt zu einer } \\
\text { Verschiebung der zuvor etablierten Therapielinien. }\end{array}$ & EK & & \\
\hline 3.76. & $\begin{array}{l}\text { Eine Systemtherapie mit einem zugelassenen Tyrosinkinase-Inhibitor soll nach Progress oder bei } \\
\text { Unverträglichkeit von Atezolizumab und Bevacizumab angeboten werden bei HCC-Patienten im } \\
\text { Child-Pugh-Stadium A und BCLC B oder C, mit Fernmetastasen oder einer Tumorlokalisation, die } \\
\text { lokoregionär nicht kontrolliert oder reseziert werden kann. }\end{array}$ & EK & & \\
\hline 3.77. & $\begin{array}{l}\text { Bei HCC-Patienten mit Tumorprogress unter einer Therapie mit Sorafenib, Child-Pugh-Stadium A } \\
\text { und ECOG } 0 \text { - } 1 \text { soll eine weitere Systemtherapie angeboten werden. Hierfür stehen die beiden } \\
\text { Tyrosinkinase-Inhibitoren Regorafenib und Cabozantinib oder bei einem Alpha-Fetoprotein-Wert } \\
\text { von } \geq 400 \mathrm{ng} / \mathrm{ml} \text { der VEGFR-2-Antikörper Ramucirumab zur Verfügung. }\end{array}$ & A & 2 & $\begin{array}{l}\text { de novo } \\
{[63-65]}\end{array}$ \\
\hline 3.78. & $\begin{array}{l}\text { Bei HCC-Patienten im Child-Pugh-Stadium A und ECOG 0-1 mit Tumorprogress unter einer Therapie } \\
\text { mit Lenvatinib soll eine weitere tumorspezifische Therapie angeboten werden. }\end{array}$ & EK & & \\
\hline 3.79 & $\begin{array}{l}\text { Die laufende Systemtherapie sollte nicht über einen radiologischen Progress hinaus fortgesetzt } \\
\text { werden. } \\
\text { Die Toxizität der Therapie sollte engmaschig überwacht und berücksichtigt werden. }\end{array}$ & EK & & \\
\hline 3.80 & $\begin{array}{l}\text { Für einzelne Immuntherapie-naive HCC-Patienten mit erhaltener Leberfunktion (im Stadium Child- } \\
\text { Pugh A), mit Fernmetastasen oder einer Tumorlokalisation, die lokoregionär nicht kontrolliert oder re- } \\
\text { seziert werden kann und für die keine zugelassene Therapie mehr zur Verfügung steht, kann entweder } \\
\text { eine Immun-Monotherapie mit den anti-PD-1-Antikörpern Nivolumab oder Pembrolizumab oder eine } \\
\text { Kombinationstherapie mit Nivolumab und dem CTLA-4-Antikörper Ipilimumab angeboten werden. }\end{array}$ & EK & & \\
\hline
\end{tabular}

\section{Sequenztherapie beim HCC innerhalb der zugelassenen Indikationen}

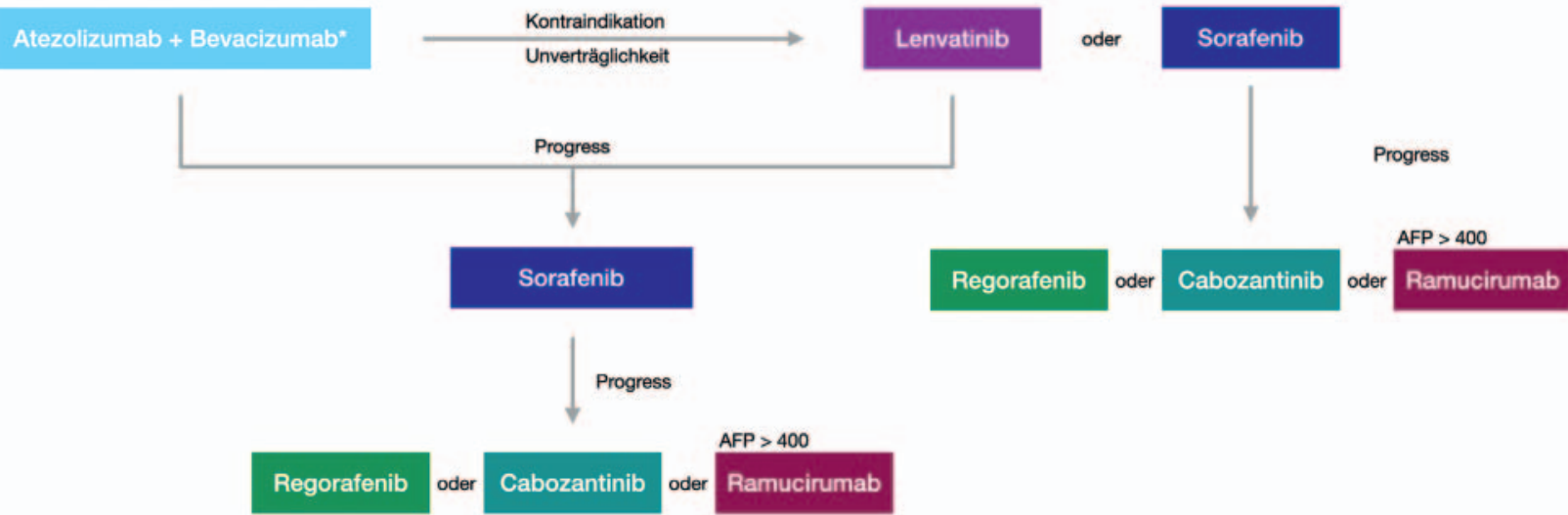

* Nach Leitlinie empfohlene Erstlinientherapie

- Abb. 4 Sequenztherapie beim HCC innerhalb der zugelassenen Indikationen. 


\begin{tabular}{|c|c|c|c|c|}
\hline Nr. & Empfehlung/Statement & EG & LoE & Quellen \\
\hline 3.81. & $\begin{array}{l}\text { Für einzelne HCC-Patienten im Child-Pugh-Stadium B (bis } 8 \text { Punkte), mit Fernmetastasen oder einer } \\
\text { Tumorlokalisation, die lokoregionär nicht kontrolliert oder reseziert werden kann, und mit einem } \\
\text { ECOG-Status von 0-1 kann eine Systemtherapie mit Sorafenib angeboten werden. }\end{array}$ & 0 & 2 & $\begin{array}{l}\text { de novo } \\
{[66-70]}\end{array}$ \\
\hline 3.82. & $\begin{array}{l}\text { Für einzelne HCC-Patienten im Child-Pugh-Stadium B (bis } 8 \text { Punkte), mit Fernmetastasen oder einer } \\
\text { Tumorlokalisation, die lokoregionär nicht kontrolliert oder reseziert werden kann, und einem } \\
\text { ECOG-Status von 0-1 kann eine Immuntherapie mit einem anti-PD-1-Antikörper angeboten } \\
\text { werden. }\end{array}$ & EK & & \\
\hline 3.83. & Bei HCC-Patienten im Stadium Child-Pugh C sollte keine Systemtherapie durchgeführt werden. & EK & & \\
\hline
\end{tabular}

3.5.5. Kombination oder Vergleich von Systemtherapie mit lokoregionärer Therapie

\begin{tabular}{|l|l|l|l|l|l|l|}
\hline Nr. & Empfehlung/Statement & Quellen \\
\hline 3.84. & $\begin{array}{l}\text { Für HCC-Patienten im Stadium Child-Pugh A oder B mit 7 Punkten und BCLC C mit überwiegendem } \\
\text { Leberbefall zeigt eine TARE keine Überlegenheit zu Sorafenib. } \\
\text { Ein Vergleich zwischen TARE und anderen medikamentösen Therapien außer Sorafenib liegt bisher } \\
\text { nicht vor. }\end{array}$ & $\begin{array}{l}\text { de novo } \\
{[55,71]}\end{array}$ \\
\hline 3.85. & $\begin{array}{l}\text { Bei HCC-Patienten im Stadium Child-Pugh A und BCLC B, die eine lokoregionäre Therapie erhalten, } \\
\text { sollte außerhalb von Studien keine zusätzliche Kombination mit einer Systemtherapie erfolgen. }\end{array}$ & A \\
\hline
\end{tabular}

\subsubsection{Adjuvante Therapie nach Resektion}

\begin{tabular}{|c|c|c|c|c|}
\hline Nr. & Empfehlung/Statement & EG & LoE & Quellen \\
\hline 3.86. & $\begin{array}{l}\text { Bei HCC-Patienten nach erfolgreicher Resektion oder lokaler Ablation mit radiologisch kompletter } \\
\text { Remission soll außerhalb von Studien keine adjuvante Systemtherapie durchgeführt werden. }\end{array}$ & A & 2 & $\begin{array}{l}\text { de novo } \\
{[72]}\end{array}$ \\
\hline
\end{tabular}

\subsubsection{Verlaufskontrollen unter Systemtherapie}

\begin{tabular}{|c|c|}
\hline Nr. & Empfehlung/Statement \\
\hline 3.87. & $\begin{array}{l}\text { Beim HCC unter Systemtherapie sollte alle } 6-12 \text { Wochen die am besten geeignete Schnittbildge- } \\
\text { bung (CT oder MRT) durchgeführt werden. Die Interpretation im klinischen Alltag sollte sich an den } \\
\text { Auswerteprinzipien von RECIST } 1.1 \text { und mRECIST sowie für Patienten unter einer Immuntherapie an } \\
\text { iRECIST orientieren. }\end{array}$ \\
\hline
\end{tabular}

\begin{tabular}{|l|l|}
\hline EG & Quellen \\
\hline EK & \\
\hline
\end{tabular}

\subsubsection{HCC bei Kindern und Jugendlichen}

\begin{tabular}{|l|l|l|l|l|l|l|}
\hline Nr. & Empfehlung/Statement & Luellen \\
\hline 3.88. & Patienten $<17$ Jahre sollen einem pädiatrisch-onkologischen Zentrum zugewiesen werden. & EK \\
\hline
\end{tabular}




\subsection{Supportivtherapie des hepatozellulären Karzinoms und der biliären Karzinome}

\subsubsection{Ernährung}

\begin{tabular}{|l|l|l|}
\hline Nr. & Empfehlung/Statement & EG \\
\hline 3.89. & $\begin{array}{l}\text { Mangelernährung beeinträchtigt die Lebensqualität und Therapietoleranz. } \\
\text { Eine Mangelernährung sollte erfasst und behandelt werden. }\end{array}$ & EK \\
\hline
\end{tabular}

\subsubsection{Palliativmedizinische Behandlung beim HCC/CCA}

\begin{tabular}{|c|c|c|c|c|}
\hline Nr. & Empfehlung/Statement & EG & LoE & Quellen \\
\hline 3.90 . & $\begin{array}{l}\text { Alle Patienten mit einer Krebserkrankung sollen unabhängig vom Krankheitsstadium Zugang } \\
\text { zu Informationen über Palliativversorgung (z. B. durch Auslage von Flyern) haben. }\end{array}$ & EK & & \\
\hline 3.91 . & $\begin{array}{l}\text { Allen Patienten soll nach der Diagnose einer nichtheilbaren Krebserkrankung eine Palliativversor- } \\
\text { gung angeboten werden, unabhängig davon, ob eine tumorspezifische Therapie durchgeführt wird. }\end{array}$ & A & 1 & $\begin{array}{l}\text { S3-Leitlinie } \\
\text { Palliativmedi- } \\
\text { zin Langversi- } \\
\text { on } 2.1 \text {-Januar } \\
2020\end{array}$ \\
\hline 3.92 . & $\begin{array}{l}\text { Allen Patienten mit einem HCC im Stadium BCLC D sollte aktiv eine Palliativversorgung angeboten } \\
\text { werden. }\end{array}$ & EK & & \\
\hline 3.93. & $\begin{array}{l}\text { Allen Patienten mit einem Cholangiokarzinom im Stadium IV nach UICC-Klassifikation soll eine } \\
\text { Palliativversorgung angeboten werden. }\end{array}$ & EK & & \\
\hline 3.94 . & Der Pruritus sollte analog der AWMF-S2k-Leitlinie Pruritus behandelt werden. & EK & & \\
\hline
\end{tabular}

\subsubsection{Rehabilitation, Sport- und Bewegungstherapie}

\begin{tabular}{|l|l|l|}
\hline Nr. & Empfehlung/Statement & EG \\
\hline 3.95. & $\begin{array}{l}\text { Patienten mit HCC/CCA sollten zu körperlichen Aktivitäten und/oder Bewegungstherapie motiviert } \\
\text { werden. }\end{array}$ & EK \\
\hline 3.96. & $\begin{array}{l}\text { Der Nutzen einer Anschlussheilbehandlung und von Rehabilitationsmaßnahmen (hinsichtlich } \\
\text { Lebensqualität, Leistungsfähigkeit, krankheitsfreiem Überleben, Gesamtüberleben) ist für das } \\
\text { HCC/CCA nicht ausreichend evaluiert. Dennoch sollte den Patienten, die die Voraussetzungen } \\
\text { erfüllen, eine AHB oder Rehabilitation angeboten werden. }\end{array}$ \\
\hline $\begin{array}{l}\text { Die rehabilitative Therapie soll medizinische, pflegerische, aufklärende, trainierende und psycho- } \\
\text { soziale Maßnahmen umfassen, die dem individuellen Rehabilitationsbedarf angepasst werden. }\end{array}$ & EK \\
\hline
\end{tabular}

\subsubsection{Psychoonkologie}

Nr.

3.98.

3.99.

\section{Empfehlung/Statement}

Die Erfassung der psychischen Belastung sowie die psychoonkologische Behandlung sollten, wie in der S3-Querschnittsleitlinie Psychoonkologie beschrieben, erfolgen.

Die Kommunikation mit Patienten mit HCC/CCA und ihren Angehörigen soll wiederholt in allen Phasen der Erkrankung und durch alle behandelnden Berufsgruppen patientenzentriert erfolgen und soll sich an deren individuellen Anliegen, Bedürfnissen und Präferenzen orientieren, welche Information, Aufklärung und Beteiligung an Entscheidungen betreffen. 


\section{Diagnostik und Therapie biliärer Karzinome}

Die S3-Leitlinie Diagnostik und Therapie des hepatozellulären Karzinoms und biliärer Karzinome umfasst 2 Tumorentitäten. Zur Vereinfachung sind die Tumorentitäten hier getrennt veröffentlicht. An dieser Stelle verweisen wir daher auf die S3-Leitlinie Diagnostik und Therapie des hepatozellulären Karzinoms.

\section{Qualitätsindikatoren}

Qualitätsindikatoren, die gemäß einer standardisierten Methodik von den starken (A) Empfehlungen dieser Leitlinie abgeleitet wurden, finden sich in der Langversion dieser Leitlinie.

\section{Abbildungsverzeichnis}

\begin{tabular}{|l|c|}
\hline Abb. 1 Diagnostikalgorithmus HCC & 95 \\
\hline Abb. 2 Therapiealgorithmus HCC in Zirrhose & 100 \\
\hline $\begin{array}{l}\text { Abb. } 3 \text { Therapiealgorithmus des HCC in Zirrhose } \\
\text { im Stadium A1 und A2 }\end{array}$ & 101 \\
\hline $\begin{array}{l}\text { Abb. } 4 \text { Sequenztherapie beim HCC innerhalb } \\
\text { der zugelassenen Indikationen }\end{array}$ & 102 \\
\hline
\end{tabular}

\section{Tabellenverzeichnis}

\begin{tabular}{|l|c|}
\hline \begin{tabular}{l} 
Tab. 1 Beteiligte Fachgesellschaften und Organisationen \\
\hline Tab. 2 Arbeitsgruppen und deren Mitglieder
\end{tabular} & 86 \\
\hline $\begin{array}{l}\text { Tab. } 3 \text { Beteiligte Experten } \\
\text { Tab. } 4 \text { Schema der Evidenzgraduierung nach Oxford } \\
\text { (Version 2011) [1] }\end{array}$ & 87 \\
\hline Tab. 5 Schema der Empfehlungsgraduierung & 91 \\
\hline Tab. 6 Konsensstärke & 92 \\
\hline $\begin{array}{l}\text { Tab. } 7 \text { Übersicht der gängigsten Selektionskriterien } \\
\text { zur Lebertransplantation }\end{array}$ & 92 \\
\hline $\begin{array}{l}\text { Tab. } 8 \text { Definitionen } \\
\end{array}$ & 96 \\
\hline
\end{tabular}

\section{Interessenkonflikt}

Die Übersicht über die Interessenkonflikte der Autorinnen und Autoren sind im Leitlinienreport veröffentlicht.

\section{Literatur}

[1] Howick J et al. The 2011 Oxford CEBM Evidence Levels of Evidence (Introductory Document). Available from: http://www.cebm.net/index. aspx?o=5653_2011

[2] Arbeitsgemeinschaft der Wissenschaftlichen Medizinischen Fachgesellschaften - Ständige Kommission, L. AWMF-Regelwerk „Leitlinien“. 1. Auflage 2012 [cited 09.12.2013]. Available from: http://www.awmf.org/ leitlinien/awmf-regelwerk/awmf-regelwerk.html
[3] Bruix ], Sherman M. Management of hepatocellular carcinoma: an update. Hepatology 2011; 53: 1020-1022

[4] Brouwer WP et al. Prediction of long-term clinical outcome in a diverse chronic hepatitis B population: Role of the PAGE-B score. J Viral Hepat 2017; 24: 1023-1031

[5] Papatheodoridis G et al. PAGE-B predicts the risk of developing hepatocellular carcinoma in Caucasians with chronic hepatitis B on 5-year antiviral therapy. J Hepatol 2016; 64: 800-806

[6] EASL Clinical Practice Guidelines: Management of hepatocellular carcinoma. J Hepatol 2018; 69: 182-236

[7] Inoue $\mathrm{M}$ et al. Influence of coffee drinking on subsequent risk of hepatocellular carcinoma: a prospective study in Japan. J Natl Cancer Inst 2005; 97 (4): 293-300

[8] Bravi F et al. Coffee and the risk of hepatocellular carcinoma and chronic liver disease: a systematic review and meta-analysis of prospective studies. Eur J Cancer Prev 2017; 26: 368-377

[9] Aleksandrova $\mathrm{K}$ et al. The association of coffee intake with liver cancer risk is mediated by biomarkers of inflammation and hepatocellular injury: data from the European Prospective Investigation into Cancer and Nutrition. Am J Clin Nutr 2015; 102: 1498-1508

[10] Setiawan VW et al. Association of coffee intake with reduced incidence of liver cancer and death from chronic liver disease in the US multiethnic cohort. Gastroenterology 2015; 148: 118-125; quiz e15

[11] Zhang BH, Yang BH, Tang ZY. Randomized controlled trial of screening for hepatocellular carcinoma. J Cancer Res Clin Oncol 2004; 130: 417422

[12] Pocha $C$ et al. Surveillance for hepatocellular cancer with ultrasonography vs. computed tomography - a randomised study. Aliment Pharmacol Ther 2013; 38: 303-312

[13] Trinchet JC et al. Ultrasonographic surveillance of hepatocellular carcinoma in cirrhosis: a randomized trial comparing 3- and 6-month periodicities. Hepatology 2011; 54: 1987-1997

[14] Tzartzeva K et al. Surveillance Imaging and Alpha Fetoprotein for Early Detection of Hepatocellular Carcinoma in Patients With Cirrhosis: A Meta-analysis. Gastroenterology 2018; 154: 1706-1718.e1

[15] Song BG et al. Additional role of liver stiffness measurement in stratifying residual hepatocellular carcinoma risk predicted by serum biomarkers in chronic hepatitis B patients under antiviral therapy. Eur J Gastroenterol Hepatol 2018; 30: 1447-1452

[16] Ioannou GN et al. Increased Risk for Hepatocellular Carcinoma Persists Up to 10 Years After HCV Eradication in Patients With Baseline Cirrhosis or High FIB-4 Scores. Gastroenterology 2019; 157: 1264-1278.e4

[17] Burrel M et al. MRI angiography is superior to helical CT for detection of HCC prior to liver transplantation: an explant correlation. Hepatology 2003; 38: 1034-1042

[18] Di Martino $\mathrm{M}$ et al. Hepatocellular carcinoma in cirrhotic patients: prospective comparison of US, CT and MR imaging. Eur Radiol 2013; 23: 887-896

[19] Haradome $\mathrm{H}$ et al. Additional value of gadoxetic acid-DTPA-enhanced hepatobiliary phase MR imaging in the diagnosis of early-stage hepatocellular carcinoma: comparison with dynamic triple-phase multidetector CT imaging. J Magn Reson Imaging 2011; 34: 69-78

[20] Khalili $\mathrm{K}$ et al. Optimization of imaging diagnosis of 1-2 cm hepatocelIular carcinoma: an analysis of diagnostic performance and resource utilization. J Hepatol 2011; 54: 723-728

[21] Chan AC et al. Evaluation of the seventh edition of the American Joint Committee on Cancer tumour-node-metastasis (TNM) staging system for patients undergoing curative resection of hepatocellular carcinoma: implications for the development of a refined staging system. HPB (Oxford) 2013; 15: 439-448 
[22] Chevret $\mathrm{S}$ et al. A new prognostic classification for predicting survival in patients with hepatocellular carcinoma. Groupe d'Etude et de Traitement du Carcinome Hepatocellulaire. J Hepatol 1999; 31: 133-141

[23] Johnson PJ et al. Assessment of liver function in patients with hepatocellular carcinoma: a new evidence-based approach-the ALBI grade. J Clin Oncol 2015; 33: 550-558

[24] Kitai $S$ et al. Validation of a new prognostic staging system for hepatocellular carcinoma: a comparison of the biomarker-combined Japan Integrated Staging Score, the conventional Japan Integrated Staging Score and the BALAD Score. Oncology 2008; 75 (Suppl. 1): 83-90

[25] Leung TW et al. Construction of the Chinese University Prognostic Index for hepatocellular carcinoma and comparison with the TNM staging system, the Okuda staging system, and the Cancer of the Liver Italian Program staging system: a study based on 926 patients. Cancer 2002; 94: $1760-1769$

[26] Marrero JA et al. Prognosis of hepatocellular carcinoma: comparison of 7 staging systems in an American cohort. Hepatology 2005; 41: 707-716

[27] Pinato DJ et al. The ALBI grade provides objective hepatic reserve estimation across each BCLC stage of hepatocellular carcinoma. J Hepatol 2017; 66: 338-346

[28] Vitale $A$ et al. Validation of the BCLC prognostic system in surgical hepatocellular cancer patients. Transplant Proc 2009; 41: 1260-1263

[29] Yau T et al. Development of Hong Kong Liver Cancer staging system with treatment stratification for patients with hepatocellular carcinoma. Gastroenterology 2014; 146: 1691-1700.e3

[30] Huang X, Lu S. Impact of preoperative locoregional therapy on recurrence and patient survival following liver transplantation for hepatocellular carcinoma: a meta-analysis. Scand J Gastroenterol 2017; 52: 143 149

[31] Kulik L et al. Therapies for patients with hepatocellular carcinoma awaiting liver transplantation: A systematic review and meta-analysis. Hepatology 2018; 67: 381-400

[32] Sneiders D et al. Systematic Review and Meta-Analysis of Posttransplant Hepatic Artery and Biliary Complications in Patients Treated With Transarterial Chemoembolization Before Liver Transplantation. Transplantation 2018; 102: 88-96

[33] Degroote $\mathrm{H}$ et al. Extended criteria for liver transplantation in hepatocellular carcinoma. A retrospective, multicentric validation study in Belgium. Surg Oncol 2019; 33: 231-238

[34] Parikh ND, Waljee AK, Singal AG. Downstaging hepatocellular carcinoma: A systematic review and pooled analysis. Liver Transpl 2015; 21: 1142-1152

[35] Bruix J et al. Adjuvant sorafenib for hepatocellular carcinoma after resection or ablation (STORM): a phase 3, randomised, double-blind, placebo-controlled trial. Lancet Oncol 2015; 16: 1344-1354

[36] Lee JH et al. Adjuvant immunotherapy with autologous cytokine-induced killer cells for hepatocellular carcinoma. Gastroenterology 2015; 148: 1383-1391.e6

[37] Mazzaferro $V$ et al. Prevention of hepatocellular carcinoma recurrence with alpha-interferon after liver resection in HCV cirrhosis. Hepatology 2006; 44: 1543-1554

[38] Samuel $\mathrm{M}$ et al. Neoadjuvant and adjuvant therapy for surgical resection of hepatocellular carcinoma. Cochrane Database Syst Rev 2009; 1: Cd001199

[39] Takayama T et al. Adoptive immunotherapy to lower postsurgical recurrence rates of hepatocellular carcinoma: a randomised trial. Lancet 2000; 356: 802-807

[40] Chen MS et al. A prospective randomized trial comparing percutaneous local ablative therapy and partial hepatectomy for small hepatocellular carcinoma. Ann Surg 2006; 243: 321-328
[41] Feng $\mathrm{K}$ et al. A randomized controlled trial of radiofrequency ablation and surgical resection in the treatment of small hepatocellular carcinoma. J Hepatol 2012; 57: 794-802

[42] Huang J et al. A randomized trial comparing radiofrequency ablation and surgical resection for HCC conforming to the Milan criteria. Ann Surg 2010; 252: 903-912

[43] Ng KKC et al. Randomized clinical trial of hepatic resection versus radiofrequency ablation for early-stage hepatocellular carcinoma. Br J Surg 2017; 104: 1775-1784

[44] Yin L et al. Partial hepatectomy vs. transcatheter arterial chemoembolization for resectable multiple hepatocellular carcinoma beyond Milan Criteria: a RCT. J Hepatol 2014; 61: 82-88

[45] Lencioni R, Llovet JM. Modified RECIST (mRECIST) assessment for hepatocellular carcinoma. Semin Liver Dis 2010; 30: 52-60

[46] Di Costanzo GG et al. Radiofrequency ablation versus laser ablation for the treatment of small hepatocellular carcinoma in cirrhosis: a randomized trial. J Gastroenterol Hepatol 2015; 30: 559-565

[47] Cucchetti A et al. An explorative data-analysis to support the choice between hepatic resection and radiofrequency ablation in the treatment of hepatocellular carcinoma. Dig Liver Dis 2014; 46: 257-263

[48] Peng ZW et al. Radiofrequency ablation with or without transcatheter arterial chemoembolization in the treatment of hepatocellular carcinoma: a prospective randomized trial. J Clin Oncol 2013; 31: 426-432

[49] Liu $\mathrm{H}$ et al. Randomized clinical trial of chemoembolization plus radiofrequency ablation versus partial hepatectomy for hepatocellular carcinoma within the Milan criteria. Br J Surg 2016; 103: 348-356

[50] Endo $\mathrm{K}$ et al. Efficacy of combination therapy with transcatheter arterial chemoembolization and radiofrequency ablation for intermediate-stage hepatocellular carcinoma. Scand J Gastroenterol 2018; 53: 1575-1583

[51] Lo CM et al. Randomized controlled trial of transarterial lipiodol chemoembolization for unresectable hepatocellular carcinoma. Hepatology 2002; 35: 1164-1171

[52] Llovet JM et al. Arterial embolisation or chemoembolisation versus symptomatic treatment in patients with unresectable hepatocellular carcinoma: a randomised controlled trial. Lancet 2002; 359: 1734-1739

[53] Golfieri R et al. Randomised controlled trial of doxorubicin-eluting beads vs conventional chemoembolisation for hepatocellular carcinoma. $\mathrm{Br}$ J Cancer 2014; 111: 255-264

[54] Lammer J et al. Prospective randomized study of doxorubicin-elutingbead embolization in the treatment of hepatocellular carcinoma: results of the PRECISION V study. Cardiovasc Intervent Radiol 2010; 33: 41-52

[55] Abdel-Rahman O, Elsaye Z. Yttrium-90 microsphere radioembolisation for unresectable hepatocellular carcinoma. Cochrane Database Syst Rev 2020; 1: Cd011313

[56] Yang Y, Si T. Yttrium-90 transarterial radioembolization versus conventional transarterial chemoembolization for patients with hepatocellular carcinoma: a systematic review and meta-analysis. Cancer Biol Med 2018; 15: 299-310

[57] Ludwig JM et al. Meta-analysis: adjusted indirect comparison of drugeluting bead transarterial chemoembolization versus (90)Y-radioembolization for hepatocellular carcinoma. Eur Radiol 2017; 27: 2031-2041

[58] Casadei Gardini A et al. Radioembolization versus chemoembolization for unresectable hepatocellular carcinoma: a meta-analysis of randomized trials. Onco Targets Ther 2018; 11: 7315-7321

[59] Finn RS et al. Atezolizumab plus Bevacizumab in Unresectable Hepatocellular Carcinoma. N Engl J Med 2020; 382: 1894-1905

[60] Cheng AL et al. Efficacy and safety of sorafenib in patients in the AsiaPacific region with advanced hepatocellular carcinoma: a phase III randomised, double-blind, placebo-controlled trial. Lancet Oncol 2009; 10: 25-34

[61] Llovet JM et al. Sorafenib in advanced hepatocellular carcinoma. N Engl J Med 2008; 359: 378-390 
[62] Kudo $\mathrm{M}$ et al. Lenvatinib versus sorafenib in first-line treatment of patients with unresectable hepatocellular carcinoma: a randomised phase 3 non-inferiority trial. The Lancet 2018; 391: 1163-1173

[63] Bruix J et al. Regorafenib for patients with hepatocellular carcinoma who progressed on sorafenib treatment (RESORCE): a randomised, doubleblind, placebo-controlled, phase 3 trial. Lancet 2017; 389: 56-66

[64] Abou-Alfa GK et al. Cabozantinib in Patients with Advanced and Progressing Hepatocellular Carcinoma. N Engl J Med 2018; 379: 54-63

[65] Zhu AX et al. Ramucirumab after sorafenib in patients with advanced hepatocellular carcinoma and increased $\alpha$-fetoprotein concentrations (REACH-2): a randomised, double-blind, placebo-controlled, phase 3 trial. Lancet Oncol 2019; 20: 282-296

[66] Marrero JA et al. Observational registry of sorafenib use in clinical practice across Child-Pugh subgroups: The GIDEON study. J Hepatol 2016; 65: 1140-1147

[67] Ganten TM et al. Sorafenib in Patients with Hepatocellular CarcinomaResults of the Observational INSIGHT Study. Clin Cancer Res 2017; 23: 5720-5728

[68] Leal CRG et al. Survival and tolerance to sorafenib in Child-Pugh B patients with hepatocellular carcinoma: a prospective study. Invest New Drugs 2018; 36: 911-918
[69] Pressiani T et al. Sorafenib in patients with Child-Pugh class A and B advanced hepatocellular carcinoma: a prospective feasibility analysis. Ann Oncol 2013; 24: 406-411

[70] Ogasawara S et al. Sorafenib treatment in Child-Pugh A and B patients with advanced hepatocellular carcinoma: safety, efficacy and prognostic factors. Invest New Drugs 2015; 33: 729-739

[71] Vilgrain $\vee$ et al. Efficacy and safety of selective internal radiotherapy with yttrium-90 resin microspheres compared with sorafenib in locally advanced and inoperable hepatocellular carcinoma (SARAH): an openlabel randomised controlled phase 3 trial. Lancet Oncol 2017; 18: 1624 1636

[72] Lencioni R et al. Sorafenib or placebo plus TACE with doxorubicin-eluting beads for intermediate stage HCC: The SPACE trial. J Hepatol 2016; 64: 1090-1098

[73] Meyer T et al. Sorafenib in combination with transarterial chemoembolisation in patients with unresectable hepatocellular carcinoma (TACE 2): a randomised placebo-controlled, double-blind, phase 3 trial. Lancet Gastroenterol Hepatol 2017; 2: 565-575

[74] Chow PKH et al. SIRveNIB: Selective Internal Radiation Therapy Versus Sorafenib in Asia-Pacific Patients With Hepatocellular Carcinoma. J Clin Oncol 2018; 36: 1913-1921 